\title{
Testing for change-points in long-range dependent time series by means of a self-normalized Wilcoxon test
}

\author{
Annika Betken* \\ Fakultät für Mathematik, Ruhr-Universität Bochum, 44780 Bochum, Germany.
}

\begin{abstract}
We propose a testing procedure based on the Wilcoxon two-sample test statistic in order to test for change-points in the mean of long-range dependent data. We show that the corresponding self-normalized test statistic converges in distribution to a non-degenerate limit under the hypothesis that no change occurred and that it diverges to infinity under the alternative of a change-point with constant height. Furthermore, we derive the asymptotic distribution of the self-normalized Wilcoxon test statistic under local alternatives, that is under the assumption that the height of the level shift decreases as the sample size increases. Regarding the finite sample performance, simulation results confirm that the self-normalized Wilcoxon test yields a consistent discrimination between hypothesis and alternative and that its empirical size is already close to the significance level for moderate sample sizes.
\end{abstract}

Keywords: change-point problem; self-normalization; long-range dependence; Wilcoxon test; nonparametric test

\section{Introduction}

We consider a data set generated by a stochastic process $\left(X_{i}\right)_{i \geq 1}$,

$$
X_{i}=\mu_{i}+\varepsilon_{i}
$$

where $\left(\mu_{i}\right)_{i \geq 1}$ are unknown constants and where $\left(\varepsilon_{i}\right)_{i \geq 1}$ is a stationary, long-range dependent (LRD, in short) process with mean zero and finite variance. In particular, we assume that

$$
\varepsilon_{i}=G\left(\xi_{i}\right), i \geq 1,
$$

where $\left(\xi_{i}\right)_{i \geq 1}$ is a stationary Gaussian process with mean 0 , variance 1 and long-range dependence, that is with autocovariance function $\rho$ satisfying

$$
\rho(k) \sim k^{-D} L(k), k \geq 1,
$$

where $0<D<1$ (referred to as long-range dependence (LRD) parameter) and where $L$ is a slowly varying function. Furthermore, we suppose that $G: \mathbb{R} \longrightarrow \mathbb{R}$ is a measurable function with $\mathrm{E}\left(G\left(\xi_{i}\right)\right)=0$.

Provided that the previous assumptions hold for the observations $X_{1}, \ldots, X_{n}$, we wish to test the hypothesis

$$
H: \mu_{1}=\ldots=\mu_{n}
$$

against the alternative

$$
A: \mu_{1}=\ldots=\mu_{k} \neq \mu_{k+1}=\ldots=\mu_{n}
$$

*annika.betken@rub.de

Research supported by Collaborative Research Center SFB 823 Statistical modeling of nonlinear dynamic processes. 
for some $k \in\{1, \ldots, n-1\}$. Within this setting the location of the change-point is unknown under the alternative. In order to motivate our choice of a change-point test, we temporarily assume that the change-point location is known, i.e. for a given $k \in\{1, \ldots, n-1\}$ we consider the alternative

$$
A_{k}: \mu_{1}=\ldots=\mu_{k} \neq \mu_{k+1}=\ldots=\mu_{n}
$$

For the test problem $\left(H, A_{k}\right)$, the Wilcoxon two-sample rank test rejects the hypothesis of no change in the mean for large absolute values of the test statistic

$$
W_{k, n}=\sum_{i=1}^{k} \sum_{j=k+1}^{n}\left(1_{\left\{X_{i} \leq X_{j}\right\}}-\frac{1}{2}\right)
$$

The Wilcoxon change-point test for the test problem $(H, A)$ is defined by reference to the test statistic $W_{k, n}$; see Dehling, Rooch and Taqqu (2013a). It rejects the hypothesis for large values of

$$
\max _{1 \leq k \leq n-1}\left|W_{k, n}\right|=\max _{1 \leq k \leq n-1}\left|\sum_{i=1}^{k} \sum_{j=k+1}^{n}\left(1_{\left\{X_{i} \leq X_{j}\right\}}-\frac{1}{2}\right)\right| .
$$

With the objective of calculating the asymptotic distribution of the Wilcoxon test statistic under the null hypothesis, Dehling, Rooch and Taqqu (2013a) consider the stochastic process

$$
W_{n}(\lambda)=\frac{1}{n d_{n}} \sum_{i=1}^{\lfloor n r\rfloor} \sum_{j=\lfloor n r\rfloor+1}^{n}\left(1_{\left\{X_{i} \leq X_{j}\right\}}-\int_{\mathbb{R}} F(x) d F(x)\right), 0 \leq \lambda \leq 1,
$$

where $d_{n}$ denotes an appropriate normalization. Assuming that $\left(X_{i}\right)_{i \geq 1}$ has a continuous marginal distribution function $F$, the asymptotic distribution of $W_{n}$ can be derived from the empirical process invariance principle of Dehling and Taqqu (1989) as shown in Dehling, Rooch and Taqqu (2013a). It turns out that both, the limit of $W_{n}$ and the normalization $d_{n}$, depend on the Hermite expansion

$$
1_{\left\{G\left(\xi_{i}\right) \leq x\right\}}-F(x)=\sum_{q=1}^{\infty} \frac{J_{q}(x)}{q !} H_{q}\left(\xi_{i}\right),
$$

where $H_{q}$ denotes the $q$-th order Hermite polynomial and where

$$
J_{q}(x)=\mathrm{E}\left(H_{q}\left(\xi_{i}\right) 1_{\left\{G\left(\xi_{i}\right) \leq x\right\}}\right) .
$$

The scaling factor $d_{n}$ is defined by

$$
d_{n}^{2}=\operatorname{Var}\left(\sum_{j=1}^{n} H_{m}\left(\xi_{j}\right)\right)
$$

where $m$ designates the Hermite rank of the class of functions $\left\{1_{\left\{G\left(\xi_{i}\right) \leq x\right\}}-F(x), x \in \mathbb{R}\right\}$ defined by

$$
m:=\min \left\{q \geq 1: J_{q}(x) \neq 0 \text { for some } x \in \mathbb{R}\right\} .
$$

Presuming the previous conditions hold and the long-range dependence parameter $D$ meets the condition $0<D<\frac{1}{m}$, the process

$$
W_{n}(\lambda)=\frac{1}{n d_{n}} \sum_{i=1}^{\lfloor n \lambda\rfloor} \sum_{j=\lfloor n \lambda\rfloor+1}^{n}\left(1_{\left\{X_{i} \leq X_{j}\right\}}-\int_{\mathbb{R}} F(x) d F(x)\right), 0 \leq \lambda \leq 1,
$$

converges in distribution to

$$
\frac{1}{m !}\left(Z_{m}(\lambda)-\lambda Z_{m}(1)\right) \int_{\mathbb{R}} J_{m}(x) d F(x), 0 \leq \lambda \leq 1,
$$


where $\left(Z_{m}(\lambda)\right)_{\lambda \in[0,1]}$ is an $m$-th order Hermite process, which is self-similar with parameter $H=1-\frac{m D}{2} \in$ $\left(\frac{1}{2}, 1\right)$. If $m=1$, the Hermite process $Z_{m}$ equals a standard fractional Brownian motion process with Hurst parameter $H=1-\frac{D}{2}$. We refer to Taqqu $(1979)$ for a general definition of the Hermite process $Z_{m}$.

An application of the continuous mapping theorem to the process $W_{n}$ yields the asymptotic distribution of the Wilcoxon change-point test. More precisely, it has been proved by Dehling, Rooch and Taqqu (2013a) that under the hypothesis of no change in the mean, the Wilcoxon test statistic

$$
\frac{1}{n d_{n}} \max _{1 \leq k \leq n-1}\left|\sum_{i=1}^{k} \sum_{j=k+1}^{n}\left(1_{\left\{X_{i} \leq X_{j}\right\}}-\frac{1}{2}\right)\right|
$$

converges in distribution to

$$
\sup _{0 \leq \lambda \leq 1}\left|\frac{1}{m !}\left(Z_{m}(\lambda)-\lambda Z_{m}(1)\right)\right|\left|\int_{\mathbb{R}} J_{m}(x) d F(x)\right| .
$$

Furthermore, Dehling, Rooch and Taqqu (2013b) investigate the asymptotic behaviour of the Wilcoxon change-point test under the alternative with the objective of determining the height of the level shift in such a way that the power of the self-normalized Wilcoxon test is non-trivial. For this purpose, they consider local alternatives defined by

$$
A_{\tau, h_{n}}: \mu_{i}= \begin{cases}\mu & \text { for } i=1, \ldots,\lfloor n \tau\rfloor \\ \mu+h_{n} & \text { for } i=\lfloor n \tau\rfloor+1, \ldots, n\end{cases}
$$

where $0<\tau<1$ and where $h_{n} \sim c \frac{d_{n}}{n}$, so that under the sequence of local alternatives $A_{\tau, h_{n}}$ the height of the level shift decreases if the sample size increases. Under the additional assumption that $G\left(\xi_{i}\right)$ has a continuous distribution function $F$ with bounded density $f$, this guarantees that under the sequence of alternatives $A_{\tau, h_{n}}$, the process

$$
\frac{1}{n d_{n}} \sum_{i=1}^{\lfloor n \lambda\rfloor} \sum_{j=\lfloor n \lambda\rfloor+1}^{n}\left(1_{\left\{X_{i} \leq X_{j}\right\}}-\frac{1}{2}\right), 0 \leq \lambda \leq 1,
$$

converges in distribution to the limit process

$$
\frac{1}{m !}\left(Z_{m}(\lambda)-\lambda Z_{m}(1)\right) \int_{\mathbb{R}} J_{m}(x) d F(x)+c \delta_{\tau}(\lambda) \int_{\mathbb{R}} f^{2}(x) d x, 0 \leq \lambda \leq 1,
$$

where $\delta_{\tau}:[0,1] \longrightarrow \mathbb{R}$ is defined by

$$
\delta_{\tau}(\lambda)= \begin{cases}\lambda(1-\tau) & \text { for } \lambda \leq \tau \\ (1-\lambda) \tau & \text { for } \lambda \geq \tau\end{cases}
$$

By another application of the continuous mapping theorem it then follows that the Wilcoxon change-point test converges in distribution to a non-degenerate limit process under the sequence of local alternatives $A_{\tau, h_{n}} ;$ see Dehling, Rooch and Taqqu (2013b).

\section{Main Results}

An application of the Wilcoxon change-point test to a given data set presupposes that the scaling factor $d_{n}$ is known. Usually this is not the case in statistical practice so that in general the Wilcoxon change-point test as proposed in Dehling, Rooch and Taqqu (2013a) depends on an unknown normalization. As an alternative we propose a normalization that only depends on the given realizations and therefore is referred to as self-normalization. The self-normalization approach we consider has originally been established in another context; see Lobato (2001). It has been extended to the change-point testing problem by Shao and Zhang (2010) in order to test for change-points in the mean of short-range dependent time series. 
These authors used the self-normalization method on the Kolmogorov-Smirnov test statistic, in doing so also taking the change-point alternative into account. Lobato as well as Shao and Zhang considered weak dependent processes only. Following the approach in Shao and Zhang an application to possibly long-range dependent processes was introduced by Shao, who established a self-normalized version of the CUSUM change-point test; see Shao (2011).

As the CUSUM test has the disadvantage of not being robust against possible outliers in the data, an extension of the self-normalization idea to the Wilcoxon test statistic leads to a change-point test that not only has the advantage of avoiding the choice of unknown parameters but also yields a robust alternative to the CUSUM test.

Given observations $X_{1}, \ldots, X_{n}$, we consider the rank statistics defined by

$$
R_{i}=\operatorname{rank}\left(X_{i}\right)=\sum_{j=1}^{n} 1_{\left\{X_{j} \leq X_{i}\right\}}
$$

for $i=1, \ldots, n$. An extension of the self-normalization approach to the Wilcoxon change-point test is based on an application of the CUSUM change-point test in terms of the rank statistics $R_{i}$. Note that due to the identity

$$
\max _{k}\left|\sum_{i=1}^{k} R_{i}-\frac{k}{n} \sum_{i=1}^{n} R_{i}\right|=\max _{k}\left|\sum_{i=1}^{k} \sum_{j=k+1}^{n}\left(1_{\left\{X_{i} \leq X_{j}\right\}}-\frac{1}{2}\right)\right|,
$$

the CUSUM test statistic of the ranks equals the Wilcoxon change-point test statistic. Instead of dividing the test statistic (which is the maximum taken among every possible outcome of the Wilcoxon two-sample rank test) by the unknown quantity $n d_{n}$ we consider a normalization factor that depends on the location of a potential change-point and which therefore is different for every possible outcome of the Wilcoxon two-sample rank test.

We define

$$
G_{n}(k)=\frac{\left|\sum_{i=1}^{k} R_{i}-\frac{k}{n} \sum_{i=1}^{n} R_{i}\right|}{\left\{\frac{1}{n} \sum_{t=1}^{k} S_{t}^{2}(1, k)+\frac{1}{n} \sum_{t=k+1}^{n} S_{t}^{2}(k+1, n)\right\}^{\frac{1}{2}}},
$$

where

$$
\begin{aligned}
& S_{t}(j, k)=\sum_{h=j}^{t}\left(R_{h}-\bar{R}_{j, k}\right), \\
& \bar{R}_{j, k}=\frac{1}{k-j+1} \sum_{t=j}^{k} R_{t} .
\end{aligned}
$$

The self-normalized Wilcoxon test rejects the hypothesis $H: \mu_{1}=\ldots=\mu_{n}$ for large values of the test statistic

$$
T_{n}\left(\tau_{1}, \tau_{2}\right)=\sup _{k \in\left[\left\lfloor n \tau_{1}\right\rfloor,\left\lfloor n \tau_{2}\right\rfloor\right]} G_{n}(k)
$$

where $0<\tau_{1}<\tau_{2}<1$.

Note that the proportion of the data that is included in the calculation of the supremum is restricted by the choice of $\tau_{1}$ and $\tau_{2}$. This is important as the choice of $\tau_{1}$ and $\tau_{2}$ influences the properties of the test. Structural breaks at the beginning or the end of a sample are hard to detect since there is a lack of information concerning the behaviour of the time series before or after a potential break point. Hence, the interval $\left[\tau_{1}, \tau_{2}\right]$ must be small enough for the critical values not to get too large on the one hand, yet large enough to include potential break points on the other hand. A common choice is $\tau_{1}=1-\tau_{2}=0.15$; see Andrews (1993).

The following theorem states the asymptotic distribution of the test statistic $T_{n}\left(\tau_{1}, \tau_{2}\right)$ under the hypothesis of no change in the mean. 
Theorem 1. Suppose that $\left(X_{i}\right)_{i \geq 1}$ is a stationary process with continuous distribution function $F$ defined by

$$
X_{i}=\mu_{i}+G\left(\xi_{i}\right)
$$

for unknown constants $\left(\mu_{i}\right)_{i \geq 1}$ and a stationary, long-range dependent Gaussian process $\left(\xi_{i}\right)_{i \geq 1}$ with mean 0 , variance 1 and $L R D$ parameter $0<D<\frac{1}{m}$, where $m$ denotes the Hermite rank of the class of functions $1_{\left\{G\left(\xi_{i}\right) \leq x\right\}}-F(x), x \in \mathbb{R}$. Moreover, assume that $\int_{\mathbb{R}} J_{m}(x) d F(x) \neq 0$ and that $G: \mathbb{R} \longrightarrow \mathbb{R}$ is a measurable function. Then, under the hypothesis of no change in the mean, it follows that $T_{n}\left(\tau_{1}, \tau_{2}\right) \stackrel{\mathcal{D}}{\longrightarrow} T\left(m, \tau_{1}, \tau_{2}\right)$, where

$$
T\left(m, \tau_{1}, \tau_{2}\right)=\sup _{\lambda \in\left[\tau_{1}, \tau_{2}\right]} \frac{\left|Z_{m}(\lambda)-\lambda Z_{m}(1)\right|}{\left\{\int_{0}^{\lambda}\left(V_{m}(r ; 0, \lambda)\right)^{2} d r+\int_{\lambda}^{1}\left(V_{m}(r ; \lambda, 1)\right)^{2} d r\right\}^{\frac{1}{2}}}
$$

with

$$
V_{m}\left(r ; r_{1}, r_{2}\right)=Z_{m}(r)-Z_{m}\left(r_{1}\right)-\frac{r-r_{1}}{r_{2}-r_{1}}\left\{Z_{m}\left(r_{2}\right)-Z_{m}\left(r_{1}\right)\right\}
$$

for $r \in\left[r_{1}, r_{2}\right], 0<r_{1}<r_{2}<1$.

As consistency under fixed alternatives is considered as a fundamental characteristic of appropriate hypothesis testing, we aim at proving Theorem 2, which implies that if there is a change-point in the mean of constant height, the empirical power of the self-normalized Wilcoxon test tends to 1 . For this purpose, we suppose that under the alternative

$$
X_{i}=\left\{\begin{array}{l}
\mu+G\left(\xi_{i}\right), i=1, \ldots, k^{*}, \\
\mu+\Delta+G\left(\xi_{i}\right), i=k^{*}+1, \ldots, n,
\end{array}\right.
$$

where $k^{*}=\lfloor n \tau\rfloor$ and $\Delta \neq 0$ is fixed.

Theorem 2. Suppose that $\left(\xi_{i}\right)_{i>1}$ is a stationary, long-range dependent Gaussian process with mean 0 , variance 1 and LRD parameter $\bar{D}$. Moreover, let $G: \mathbb{R} \longrightarrow \mathbb{R}$ be a measurable function and assume that $G\left(\xi_{i}\right)$ has a continuous distribution function $F$. Given that the parameter $D$ satisfies $0<D<\frac{1}{m}$, where $m$ denotes the Hermite rank of the class of functions $1_{\left\{G\left(\xi_{i}\right) \leq x\right\}}-F(x), x \in \mathbb{R}, T_{n}\left(\tau_{1}, \tau_{2}\right)$ diverges in probability to $\infty$ under fixed alternatives, i.e. if $\left(X_{i}\right)_{i \geq 1}$ satisfies (2).

Furthermore, we wish to study the asymptotic behaviour of the self-normalized Wilcoxon change-point test under local alternatives defined by

$$
A_{\tau, h_{n}}(n): \mu_{i}= \begin{cases}\mu & \text { for } i=1, \ldots,\lfloor n \tau\rfloor, \\ \mu+h_{n} & \text { for } i=\lfloor n \tau\rfloor+1, \ldots, n,\end{cases}
$$

where $0<\tau<1$ and $h_{n} \longrightarrow 0$. The following theorem confirms that the self-normalized Wilcoxon test statistic converges to a non-degenerate limit under the sequence of local alternatives $A_{\tau, h_{n}}$.

Theorem 3. Suppose that $\left(\xi_{i}\right)_{i \geq 1}$ is a stationary Gaussian process with mean 0 , variance 1 and autocovariance function

$$
\rho(k) \sim k^{-D} L(k)
$$

where $L$ is a slowly varying function and where $0<D<\frac{1}{m}$. Moreover, let $G: \mathbb{R} \longrightarrow \mathbb{R}$ be a measurable function. We assume that $G\left(\xi_{i}\right)$ has a continuous distribution function $F$ with bounded density $f$. Let $m$ denote the Hermite rank of the class of functions $1_{\left\{G\left(\xi_{i}\right) \leq x\right\}}-F(x), x \in \mathbb{R}$, and suppose that $\int_{\mathbb{R}} J_{m}(x) d F(x) \neq 0$. Then, under the sequence of alternatives $A_{\tau, h_{n}}$ with $h_{n} \sim c \frac{d_{n}}{n}$, it follows that $T_{n}\left(\tau_{1}, \tau_{2}\right)$ converges in distribution to

$$
T\left(m, \tau_{1}, \tau_{2}\right)=\sup _{\lambda \in\left[\tau_{1}, \tau_{2}\right]} \frac{\left|\frac{1}{m !} \int_{\mathbb{R}} J_{m}(x) d F(x)\left(Z_{m}(\lambda)-\lambda Z_{m}(1)\right)+c \delta_{\tau}(\lambda) \int_{\mathbb{R}} f^{2}(x) d x\right|}{\left\{\int_{0}^{\lambda}\left(V_{m, \tau}(r ; 0, \lambda)\right)^{2} d r+\int_{\lambda}^{1}\left(V_{m, \tau}(r ; \lambda, 1)\right)^{2} d r\right\}^{\frac{1}{2}}},
$$


where

$$
\begin{aligned}
V_{m, \tau}(r ; 0, \lambda)= & \frac{1}{m !} \int_{\mathbb{R}} J_{m}(x) d F(x)\left(Z_{m}(r)-\frac{r}{\lambda} Z_{m}(\lambda)\right)+c \int_{\mathbb{R}} f^{2}(x) d x\left(\delta_{\tau}(r)-\frac{r}{\lambda} \delta_{\tau}(\lambda)\right), \\
V_{m, \tau}(r ; \lambda, 1)= & \frac{1}{m !} \int_{\mathbb{R}} J_{m}(x) d F(x)\left\{Z_{m}(r)-Z_{m}(\lambda)-\frac{r-\lambda}{1-\lambda}\left(Z_{m}(1)-Z_{m}(\lambda)\right)\right\} \\
& +c \int_{\mathbb{R}} f^{2}(x) d x\left(\delta_{\tau}(r)-\frac{1-r}{1-\lambda} \delta_{\tau}(\lambda)\right) .
\end{aligned}
$$

\section{Simulation studies}

We will now investigate the finite sample performance of the self-normalized Wilcoxon test statistic. For this purpose, we take $G(t)=t$ so that $\left(X_{i}\right)_{i>1}$ is a Gaussian process. Since $G$ is strictly increasing, the Hermite coefficient $J_{1}(x)$ is not equal to 0 for all $x \in \mathbb{R}$; see Dehling, Rooch and Taqqu (2013a). Therefore, it holds that $m=1$, where $m$ denotes the Hermite rank of $1_{\left\{G\left(\xi_{i}\right) \leq x\right\}}-F(x), x \in \mathbb{R}$. As a result, $T_{n}\left(\tau_{1}, \tau_{2}\right)$ has approximately the same distribution as

$$
\begin{aligned}
& \sup _{\lambda \in\left[\tau_{1}, \tau_{2}\right]} \frac{\left|B_{H}(\lambda)-\lambda B_{H}(1)\right|}{\left\{\int_{0}^{\lambda}\left(V_{H}(r ; 0, \lambda)\right)^{2} d r+\int_{\lambda}^{1}\left(V_{H}(r ; \lambda, 1)\right)^{2} d r\right\}^{\frac{1}{2}}} \\
& V_{H}\left(r ; r_{1}, r_{2}\right)=B_{H}(r)-B_{H}\left(r_{1}\right)-\frac{r-r_{1}}{r_{2}-r_{1}}\left\{B_{H}\left(r_{2}\right)-B_{H}\left(r_{1}\right)\right\},
\end{aligned}
$$

where $B_{H}$ is a fractional Brownian motion process with Hurst parameter $H=1-\frac{D}{2}$.

We set critical values on the basis of 10,000 simulations of fractional Brownian motion time series for different Hurst parameters $H$ and different levels of significance; see Table 1 .

\begin{tabular}{rrrr} 
& $10 \%$ & $5 \%$ & $1 \%$ \\
\hline$H=0.6$ & 6.182835 & 7.276568 & 9.785915 \\
$H=0.7$ & 6.847260 & 8.190125 & 11.380584 \\
$H=0.8$ & 7.767277 & 9.495194 & 13.021080 \\
$H=0.9$ & 8.520039 & 10.333602 & 14.544094
\end{tabular}

Table 1: Simulated critical values for the distribution of $T\left(1, \tau_{1}, \tau_{2}\right)$ when $\left[\tau_{1}, \tau_{2}\right]=[0.15,0.85]$. The sample size is 1000 , the number of replications is 10,000 .

The calculation of the relative frequency of false rejections under the hypothesis is based on 10,000 realizations of fractional Gaussian noise time series with varying length; see Table 2

\begin{tabular}{rcccc}
$\mathrm{n}$ & $\mathrm{H}=0.6$ & $\mathrm{H}=0.7$ & $\mathrm{H}=0.8$ & $\mathrm{H}=0.9$ \\
\hline 10 & 0.057 & 0.052 & 0.036 & 0.026 \\
50 & 0.048 & 0.050 & 0.046 & 0.052 \\
100 & 0.049 & 0.055 & 0.050 & 0.053 \\
500 & 0.053 & 0.050 & 0.049 & 0.054 \\
1000 & 0.053 & 0.053 & 0.050 & 0.052
\end{tabular}

Table 2: Level of the self-normalized Wilcoxon change-point test for fractional Gaussian noise time series of length $n$ with Hurst parameter $H$. The level of significance is $5 \%$. The calculations are based on 10,000 simulation runs.

The simulation results suggest that the self-normalized Wilcoxon test performs well under the hypothesis since empirical size and asymptotic significance level are already close for moderate sample sizes. In particular, it is notable that the size of the self-normalized Wilcoxon change-point test differs considerably from the size of the original Wilcoxon change-point test when $H=0.9$, that means when we have very strong dependence. In that case, the convergence of the Wilcoxon change-point test statistic appears to 


\begin{tabular}{|c|c|c|c|c|c|c|c|}
\hline & & \multicolumn{2}{|c|}{$\Delta=0.5$} & \multicolumn{2}{|c|}{$\Delta=1$} & \multicolumn{2}{|c|}{$\Delta=2$} \\
\hline & & $10 \%$ & $5 \%$ & $10 \%$ & $5 \%$ & $10 \%$ & $5 \%$ \\
\hline \multirow[t]{2}{*}{$H=0.6$} & $n=100$ & 0.474 & 0.348 & 0.956 & 0.916 & 1.000 & 1.000 \\
\hline & $n=500$ & 0.941 & 0.898 & 1.000 & 1.000 & 1.000 & 1.000 \\
\hline \multirow[t]{2}{*}{$H=0.7$} & $n=100$ & 0.355 & 0.239 & 0.801 & 0.696 & 1.000 & 0.999 \\
\hline & $n=500$ & 0.655 & 0.530 & 0.992 & 0.985 & 1.000 & 1.000 \\
\hline \multirow[t]{2}{*}{$H=0.8$} & $n=100$ & 0.281 & 0.177 & 0.652 & 0.526 & 0.993 & 0.984 \\
\hline & $n=500$ & 0.405 & 0.297 & 0.872 & 0.802 & 1.000 & 1.000 \\
\hline \multirow[t]{2}{*}{$H=0.9$} & $n=100$ & 0.426 & 0.287 & 0.744 & 0.628 & 0.992 & 0.983 \\
\hline & $n=500$ & 0.412 & 0.280 & 0.786 & 0.689 & 0.998 & 0.997 \\
\hline
\end{tabular}

Table 3: Empirical power of the self-normalized Wilcoxon change-point test for fractional Gaussian noise of length $n=100$ and $n=500$ with Hurst parameter $H$ and a level shift in the mean of height $\Delta$ after a proportion $\tau=0.5$. The calculations are based on 5,000 simulation runs.

be rather slow under the hypothesis (see Dehling, Rooch and Taqqu (2013a), Table 2), whereas the size of the self-normalized Wilcoxon change-point test is still close to the corresponding level of significance.

We consider fractional Gaussian noise time series with a level shift of height $\Delta$ after a proportion $\tau$ of the data in order to analyse the behaviour of the test statistic under the alternative. We have done so for several choices of $\Delta$ and $\tau$ and for sample sizes $n=100$ and $n=500$.

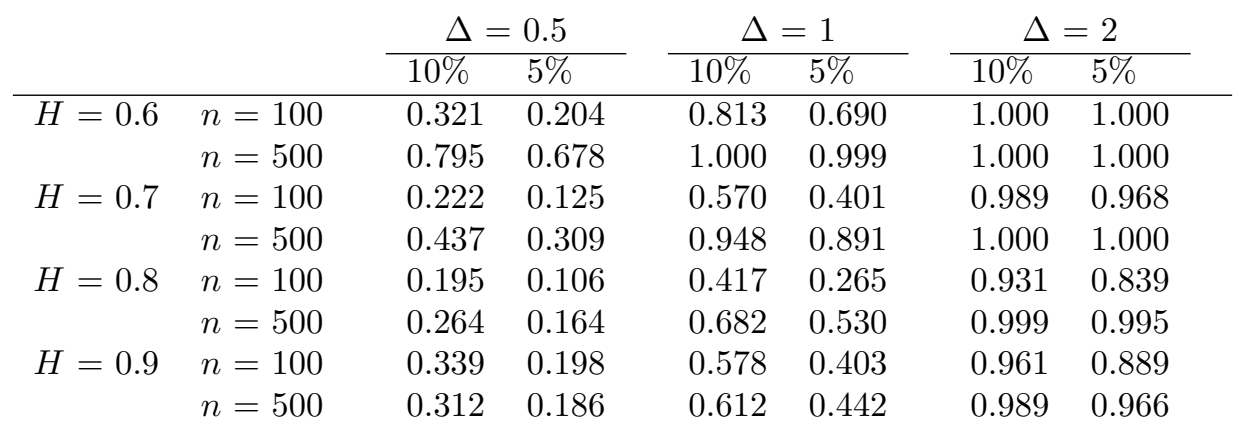

Table 4: Empirical power of the self-normalized Wilcoxon change-point test for fractional Gaussian noise of length $n=100$ and $n=500$ with Hurst parameter $H$ and a level shift in the mean of height $\Delta$ after a proportion $\tau=0.25$. The calculations are based on 5,000 simulation runs.

The simulations of the empirical power confirm that the rejection rate becomes higher when $\Delta$ increases. Comparing the empirical power for different Hurst parameters $H$, we note that the test tends to have less power as $H$ becomes large. This seems natural since when there is very strong dependence, i.e. $H$ is large, the variance of the series increases, so that it becomes harder to detect a level shift of a fixed height. In addition, change-points that are located in the middle of the sample are detected more often than change-points that are located close to the boundary of the testing region determined by $\left[\tau_{1}, \tau_{2}\right]$. Furthermore, Table 4 and Table 3 show that an increasing sample size goes along with an increase of the empirical power. This result confirms that the self-normalized Wilcoxon change-point test yields a consistent discrimination between hypothesis and alternative. 


\section{Proofs}

In order to simplify notation, we write

$$
\begin{aligned}
J(x) & =\frac{1}{m !} J_{m}(x), \\
Z(\lambda) & =Z_{m}(\lambda) .
\end{aligned}
$$

Proof of Theorem 1. The essential step in the proof of Theorem 1 is to find a representation for the test statistic $T_{n}\left(\tau_{1}, \tau_{2}\right)$ as a functional of the Wilcoxon process

$$
W_{n}(\lambda)=\frac{1}{n d_{n}} \sum_{i=1}^{\lfloor n \lambda\rfloor} \sum_{j=\lfloor n \lambda\rfloor+1}^{n}\left(1_{\left\{X_{i} \leq X_{j}\right\}}-\frac{1}{2}\right), 0 \leq \lambda \leq 1 .
$$

For this purpose, rewrite

$$
\begin{aligned}
G_{n}(k)= & \mid \frac{\left|\sum_{i=1}^{k} \sum_{j=k+1}^{n}\left(1_{\left\{X_{i} \leq X_{j}\right\}}-\frac{1}{2}\right)\right|}{\left\{\frac{1}{n} \sum_{t=1}^{k} S_{t}^{2}(1, k)+\frac{1}{n} \sum_{t=k+1}^{n} S_{t}^{2}(k+1, n)\right\}^{\frac{1}{2}}} \\
= & \frac{\frac{1}{n d_{n}}\left|\sum_{i=1}^{k} \sum_{j=k+1}^{n}\left(1_{\left\{X_{i} \leq X_{j}\right\}}-\frac{1}{2}\right)\right|}{\frac{1}{n d_{n}}\left\{\frac{1}{n} \sum_{t=1}^{k} S_{t}^{2}(1, k)+\frac{1}{n} \sum_{t=k+1}^{n} S_{t}^{2}(k+1, n)\right\}^{\frac{1}{2}}} .
\end{aligned}
$$

As we have

$$
\frac{1}{n d_{n}}\left|\sum_{i=1}^{k} \sum_{j=k+1}^{n}\left(1_{\left\{X_{i} \leq X_{j}\right\}}-\frac{1}{2}\right)\right|=\left|W_{n}(\lambda)\right|
$$

for the numerator of $G_{n}(k)$ if $k=\lfloor n \lambda\rfloor$, it remains to show that the denominator of $G_{n}(k)$ can be represented as a functional of $W_{n}$. Since

$$
R_{i}=n+1-\sum_{j=1}^{n} 1_{\left\{X_{i} \leq X_{j}\right\}}
$$

almost surely, it follows that

$$
\begin{aligned}
S_{t}(1, k)= & -\sum_{h=1}^{t}\left(\sum_{j=1}^{n} 1_{\left\{X_{h} \leq X_{j}\right\}}-\frac{1}{k} \sum_{i=1}^{k} \sum_{j=1}^{n} 1_{\left\{X_{i} \leq X_{j}\right\}}\right) \\
= & -\left\{\sum_{i=1}^{t} \sum_{j=t+1}^{n}\left(1_{\left\{X_{i} \leq X_{j}\right\}}-\frac{1}{2}\right)+\sum_{i=1}^{t} \sum_{j=1}^{t}\left(1_{\left\{X_{i} \leq X_{j}\right\}}-\frac{1}{2}\right)\right. \\
& \left.-\frac{t}{k} \sum_{i=1}^{k} \sum_{j=k+1}^{n}\left(1_{\left\{X_{i} \leq X_{j}\right\}}-\frac{1}{2}\right)-\frac{t}{k} \sum_{i=1}^{k} \sum_{j=1}^{k}\left(1_{\left\{X_{i} \leq X_{j}\right\}}-\frac{1}{2}\right)\right\}
\end{aligned}
$$

almost surely. Moreover, it is well known that

$$
\sum_{i=1}^{l} \sum_{j=1}^{l} 1_{\left\{X_{i} \leq X_{j}\right\}}=\frac{l(l+1)}{2} .
$$

Hence,

$$
\sum_{i=1}^{l} \sum_{j=1}^{l}\left(1_{\left\{X_{i} \leq X_{j}\right\}}-\frac{1}{2}\right)=\frac{l(l+1)}{2}-\frac{l^{2}}{2}=\frac{l}{2},
$$


so that

$$
\begin{aligned}
S_{t}(1, k) & =-\left\{\sum_{i=1}^{t} \sum_{j=t+1}^{n}\left(1_{\left\{X_{i} \leq X_{j}\right\}}-\frac{1}{2}\right)+\frac{t}{2}-\frac{t}{k} \sum_{i=1}^{k} \sum_{j=k+1}^{n}\left(1_{\left\{X_{i} \leq X_{j}\right\}}-\frac{1}{2}\right)-\frac{t}{k} \frac{k}{2}\right\} \\
& =-\left\{\sum_{i=1}^{t} \sum_{j=t+1}^{n}\left(1_{\left\{X_{i} \leq X_{j}\right\}}-\frac{1}{2}\right)-\frac{t}{k} \sum_{i=1}^{k} \sum_{j=k+1}^{n}\left(1_{\left\{X_{i} \leq X_{j}\right\}}-\frac{1}{2}\right)\right\}
\end{aligned}
$$

almost surely. Thus, if $\lambda \in\left[\tau_{1}, \tau_{2}\right]$,

$$
\begin{aligned}
& \int_{0}^{\lambda}\left(\sum_{i=1}^{\lfloor n r\rfloor} \sum_{j=\lfloor n r\rfloor+1}^{n}\left(1_{\left\{X_{i} \leq X_{j}\right\}}-\frac{1}{2}\right)-\frac{\lfloor n r\rfloor}{\lfloor n \lambda\rfloor} \sum_{i=1}^{\lfloor n \lambda\rfloor} \sum_{j=\lfloor n \lambda\rfloor+1}^{n}\left(1_{\left\{X_{i} \leq X_{j}\right\}}-\frac{1}{2}\right)\right)^{2} d r \\
& =\sum_{t=0}^{\lfloor n \lambda\rfloor} \int_{\frac{t}{n}}^{\frac{t+1}{n}}\left(\sum_{i=1}^{\lfloor n r\rfloor} \sum_{j=\lfloor n r\rfloor+1}^{n}\left(1_{\left\{X_{i} \leq X_{j}\right\}}-\frac{1}{2}\right)-\frac{\lfloor n r\rfloor}{\lfloor n \lambda\rfloor} \sum_{i=1}^{\lfloor n \lambda\rfloor} \sum_{j=\lfloor n \lambda\rfloor+1}^{n}\left(1_{\left\{X_{i} \leq X_{j}\right\}}-\frac{1}{2}\right)\right)^{2} d r \\
& \quad-\int_{\lambda}^{\frac{\lfloor n \lambda\rfloor+1}{n}}\left(\sum_{i=1}^{\lfloor n r\rfloor} \sum_{j=\lfloor n r\rfloor+1}^{n}\left(1_{\left\{X_{i} \leq X_{j}\right\}}-\frac{1}{2}\right)-\frac{\lfloor n r\rfloor}{\lfloor n \lambda\rfloor} \sum_{i=1}^{\lfloor n \lambda\rfloor} \sum_{j=\lfloor n \lambda\rfloor+1}^{n}\left(1_{\left\{X_{i} \leq X_{j}\right\}}-\frac{1}{2}\right)\right)^{2} d r
\end{aligned}
$$

where

$$
\sum_{i=1}^{\lfloor n r\rfloor} \sum_{j=\lfloor n r\rfloor+1}^{n}\left(1_{\left\{X_{i} \leq X_{j}\right\}}-\frac{1}{2}\right)-\frac{\lfloor n r\rfloor}{\lfloor n \lambda\rfloor} \sum_{i=1}^{\lfloor n \lambda\rfloor} \sum_{j=\lfloor n \lambda\rfloor+1}^{n}\left(1_{\left\{X_{i} \leq X_{j}\right\}}-\frac{1}{2}\right)=0
$$

for $r \in\left[\lambda, \frac{\lfloor n \lambda\rfloor+1}{n}\right)$. Therefore, the integral over that interval equals 0 . Consequently,

$$
\begin{aligned}
& \int_{0}^{\lambda}\left(\sum_{i=1}^{\lfloor n r\rfloor} \sum_{j=\lfloor n r\rfloor+1}^{n}\left(1_{\left\{X_{i} \leq X_{j}\right\}}-\frac{1}{2}\right)-\frac{\lfloor n r\rfloor}{\lfloor n \lambda\rfloor} \sum_{i=1}^{\lfloor n \lambda\rfloor} \sum_{j=\lfloor n \lambda\rfloor+1}^{n}\left(1_{\left\{X_{i} \leq X_{j}\right\}}-\frac{1}{2}\right)\right)^{2} d r \\
& =\sum_{t=0}^{\lfloor n \lambda\rfloor} \int_{\frac{t}{n}}^{\frac{t+1}{n}}\left(\sum_{i=1}^{\lfloor n r\rfloor} \sum_{j=\lfloor n r\rfloor+1}^{n}\left(1_{\left\{X_{i} \leq X_{j}\right\}}-\frac{1}{2}\right)-\frac{\lfloor n r\rfloor}{\lfloor n \lambda\rfloor} \sum_{i=1}^{\lfloor n \lambda\rfloor} \sum_{j=\lfloor n \lambda\rfloor+1}^{n}\left(1_{\left\{X_{i} \leq X_{j}\right\}}-\frac{1}{2}\right)\right)^{2} d r \\
& =\frac{1}{n} \sum_{t=0}^{k}\left(\sum_{i=1}^{t} \sum_{j=t+1}^{n}\left(1_{\left\{X_{i} \leq X_{j}\right\}}-\frac{1}{2}\right)-\frac{t}{k} \sum_{i=1}^{k} \sum_{j=k+1}^{n}\left(1_{\left\{X_{i} \leq X_{j}\right\}}-\frac{1}{2}\right)\right)^{2} \\
& =\frac{1}{n} \sum_{t=1}^{k} S_{t}^{2}(1, k)
\end{aligned}
$$

almost surely in case $k=\lfloor n \lambda\rfloor$.

For the second term in the denominator of $G_{n}(k)$ the following equations hold almost surely

$$
\begin{aligned}
S_{t}(k+1, n)= & -\left\{\sum_{h=k+1}^{t}\left(\sum_{j=1}^{n} 1_{\left\{X_{h} \leq X_{j}\right\}}-\frac{1}{n-k} \sum_{i=k+1}^{n} \sum_{j=1}^{n} 1_{\left\{X_{i} \leq X_{j}\right\}}\right)\right\} \\
= & -\left\{\sum_{i=1}^{t} \sum_{j=t+1}^{n}\left(1_{\left\{X_{i} \leq X_{j}\right\}}-\frac{1}{2}\right)+\sum_{i=1}^{t} \sum_{j=1}^{t}\left(1_{\left\{X_{i} \leq X_{j}\right\}}-\frac{1}{2}\right)\right. \\
& -\sum_{i=1}^{k} \sum_{j=k+1}^{n}\left(1_{\left\{X_{i} \leq X_{j}\right\}}-\frac{1}{2}\right)-\sum_{i=1}^{k} \sum_{j=1}^{k}\left(1_{\left\{X_{i} \leq X_{j}\right\}}-\frac{1}{2}\right) \\
& \left.-\frac{t-k}{n-k} \sum_{i=k+1}^{n} \sum_{j=1}^{k}\left(1_{\left\{X_{i} \leq X_{j}\right\}}-\frac{1}{2}\right)-\frac{t-k}{n-k} \sum_{i=k+1}^{n} \sum_{j=k+1}^{n}\left(1_{\left\{X_{i} \leq X_{j}\right\}}-\frac{1}{2}\right)\right\} .
\end{aligned}
$$


By (3) we get

$$
\sum_{i=k+1}^{n} \sum_{j=k+1}^{n}\left(1_{\left\{X_{i} \leq X_{j}\right\}}-\frac{1}{2}\right)=\frac{(n-k)(n-k+1)}{2}-\frac{(n-k)^{2}}{2}=\frac{n-k}{2} .
$$

Furthermore,

$$
1_{\left\{X_{i} \leq X_{j}\right\}}-\frac{1}{2}=1-1_{\left\{X_{j}<X_{i}\right\}}-\frac{1}{2}=-\left(1_{\left\{X_{j} \leq X_{i}\right\}}-\frac{1}{2}\right)
$$

almost surely if $i \neq j$. This yields

$$
\begin{aligned}
S_{t}(k+1, n)= & -\left\{\sum_{i=1}^{t} \sum_{j=t+1}^{n}\left(1_{\left\{X_{i} \leq X_{j}\right\}}-\frac{1}{2}\right)+\frac{t}{2}-\sum_{i=1}^{k} \sum_{j=k+1}^{n}\left(1_{\left\{X_{i} \leq X_{j}\right\}}-\frac{1}{2}\right)-\frac{k}{2}\right. \\
& \left.-\frac{t-k}{n-k} \sum_{i=k+1}^{n} \sum_{j=1}^{k}\left(1_{\left\{X_{i} \leq X_{j}\right\}}-\frac{1}{2}\right)-\frac{t-k}{n-k} \frac{n-k}{2}\right\} \\
= & -\left\{\sum_{i=1}^{t} \sum_{j=t+1}^{n}\left(1_{\left\{X_{i} \leq X_{j}\right\}}-\frac{1}{2}\right)-\frac{n-t}{n-k} \sum_{i=1}^{k} \sum_{j=k+1}^{n}\left(1_{\left\{X_{i} \leq X_{j}\right\}}-\frac{1}{2}\right)\right\} .
\end{aligned}
$$

We obtain for $\lambda \in\left[\tau_{1}, \tau_{2}\right]$

$$
\begin{aligned}
& \int_{\lambda}^{1}\left(\sum_{i=1}^{\lfloor n r\rfloor} \sum_{j=\lfloor n r\rfloor+1}^{n}\left(1_{\left\{X_{i} \leq X_{j}\right\}}-\frac{1}{2}\right)-\frac{n-\lfloor n r\rfloor}{n-\lfloor n \lambda\rfloor} \sum_{i=1}^{\lfloor n \lambda\rfloor} \sum_{j=\lfloor n \lambda\rfloor+1}^{n}\left(1_{\left\{X_{i} \leq X_{j}\right\}}-\frac{1}{2}\right)\right)^{2} d r \\
& =\sum_{t=\lfloor n \lambda\rfloor+1}^{n-1} \int_{\frac{t}{n}}^{\frac{t+1}{n}}\left(\sum_{i=1}^{\lfloor n r\rfloor} \sum_{j=\lfloor n r\rfloor+1}^{n}\left(1_{\left\{X_{i} \leq X_{j}\right\}}-\frac{1}{2}\right)-\frac{n-\lfloor n r\rfloor}{n-\lfloor n \lambda\rfloor} \sum_{i=1}^{\lfloor n \lambda\rfloor} \sum_{j=\lfloor n \lambda\rfloor+1}^{n}\left(1_{\left\{X_{i} \leq X_{j}\right\}}-\frac{1}{2}\right)\right)^{2} d r \\
& \quad+\int_{\lambda}^{\frac{\lfloor n \lambda\rfloor+1}{n}}\left(\sum_{i=1}^{\lfloor n r\rfloor} \sum_{j=\lfloor n r\rfloor+1}^{n}\left(1_{\left\{X_{i} \leq X_{j}\right\}}-\frac{1}{2}\right)-\frac{n-\lfloor n r\rfloor}{n-\lfloor n \lambda\rfloor} \sum_{i=1}^{\lfloor n \lambda\rfloor} \sum_{j=\lfloor n \lambda\rfloor+1}^{n}\left(1_{\left\{X_{i} \leq X_{j}\right\}}-\frac{1}{2}\right)\right)^{2} d r
\end{aligned}
$$

almost surely, where

$$
\sum_{i=1}^{\lfloor n r\rfloor} \sum_{j=\lfloor n r\rfloor+1}^{n}\left(1_{\left\{X_{i} \leq X_{j}\right\}}-\frac{1}{2}\right)-\frac{n-\lfloor n r\rfloor}{n-\lfloor n \lambda\rfloor} \sum_{i=1}^{\lfloor n \lambda\rfloor} \sum_{j=\lfloor n \lambda\rfloor+1}^{n}\left(1_{\left\{X_{i} \leq X_{j}\right\}}-\frac{1}{2}\right)=0
$$

if $r \in\left[\lambda, \frac{\lfloor n \lambda\rfloor+1}{n}\right)$. Therefore, the integral over that interval equals 0. For $k=\lfloor n \lambda\rfloor$ this implies

$$
\begin{aligned}
& \int_{\lambda}^{1}\left(\sum_{i=1}^{\lfloor n r\rfloor} \sum_{j=\lfloor n r\rfloor+1}^{n}\left(1_{\left\{X_{i} \leq X_{j}\right\}}-\frac{1}{2}\right)-\frac{n-\lfloor n r\rfloor}{n-\lfloor n \lambda\rfloor} \sum_{i=1}^{\lfloor n \lambda\rfloor} \sum_{j=\lfloor n \lambda\rfloor+1}^{n}\left(1_{\left\{X_{i} \leq X_{j}\right\}}-\frac{1}{2}\right)\right)^{2} d r \\
& =\frac{1}{n} \sum_{t=k+1}^{n-1}\left(\sum_{i=1}^{t} \sum_{j=t+1}^{n}\left(1_{\left\{X_{i} \leq X_{j}\right\}}-\frac{1}{2}\right)-\frac{n-t}{n-k} \sum_{i=1}^{k} \sum_{j=k+1}^{n}\left(1_{\left\{X_{i} \leq X_{j}\right\}}-\frac{1}{2}\right)\right)^{2} \\
& =\frac{1}{n} \sum_{t=k+1}^{n-1} S_{t}^{2}(k+1, n) \\
& =\frac{1}{n} \sum_{t=k+1}^{n} S_{t}^{2}(k+1, n) .
\end{aligned}
$$

Due to the previous considerations, the properly normalized denominator of $G_{n}(k)$ can (almost surely) be represented as follows

$$
\frac{1}{n d_{n}}\left\{\frac{1}{n} \sum_{t=1}^{k} S_{t}^{2}(1, k)+\frac{1}{n} \sum_{t=k+1}^{n} S_{t}^{2}(k+1, n)\right\}^{\frac{1}{2}}
$$




$$
=\left\{\int_{0}^{\lambda}\left(W_{n}(r)-\frac{c_{n}(r)}{c_{n}(\lambda)} W_{n}(\lambda)\right)^{2} d r+\int_{\lambda}^{1}\left(W_{n}(r)-\frac{1-c_{n}(r)}{1-c_{n}(\lambda)} W_{n}(\lambda)\right)^{2} d r\right\}^{\frac{1}{2}},
$$

where $c_{n}(\lambda)=\frac{\lfloor n \lambda\rfloor}{n}$ for $\lambda \in[0,1]$. All in all, this yields

$$
T_{n}\left(\tau_{1}, \tau_{2}\right)=\sup _{\lambda \in\left[\tau_{1}, \tau_{2}\right]} \frac{\left|W_{n}(\lambda)\right|}{\left\{\int_{0}^{\lambda}\left(W_{n}(r)-\frac{c_{n}(r)}{c_{n}(\lambda)} W_{n}(\lambda)\right)^{2} d r+\int_{\lambda}^{1}\left(W_{n}(r)-\frac{1-c_{n}(r)}{1-c_{n}(\lambda)} W_{n}(\lambda)\right)^{2} d r\right\}^{\frac{1}{2}}}
$$

The foregoing characterization of the self-normalized Wilcoxon test statistic points out that a representation of $T_{n}\left(\tau_{1}, \tau_{2}\right)$ as a functional of the process

$$
W_{n}(\lambda)=\frac{1}{n d_{n}} \sum_{i=1}^{\lfloor n \lambda\rfloor} \sum_{j=\lfloor n \lambda\rfloor+1}^{n}\left(1_{\left\{X_{i} \leq X_{j}\right\}}-\frac{1}{2}\right), 0 \leq \lambda \leq 1,
$$

also depends on the function series $\left(c_{n}\right)_{n \in \mathbb{N}}$ in $D[0,1]$ defined by $c_{n}(\lambda)=\frac{\lfloor n \lambda\rfloor}{n}, 0 \leq \lambda \leq 1$. Since

$$
\sup _{\lambda \in[0,1]}\left|\frac{\lfloor n \lambda\rfloor}{n}-\lambda\right|=\sup _{\lambda \in[0,1]}\left(\lambda-\frac{\lfloor n \lambda\rfloor}{n}\right) \leq \sup _{\lambda \in[0,1]}\left(\lambda-\frac{n \lambda-1}{n}\right)=\frac{1}{n} \longrightarrow 0,
$$

the sequence $c_{n}, n \in \mathbb{N}$, converges with respect to the supremum norm to $c \in D[0,1]$ defined by $c(\lambda)=\lambda$ for $\lambda \in[0,1]$. To simplify subsequent calculations, we treat $c_{n}$ and $c$ as random variables with values in the closure of

$$
M=\left\{f \in D[0,1] \mid f(\lambda)=\frac{\lfloor n \lambda\rfloor}{n} \text { for some } n \in \mathbb{N}, n \geq \frac{1}{\tau_{1}}\right\} .
$$

Note that

$$
h_{n}=\left(\begin{array}{c}
c_{n} \\
W_{n}
\end{array}\right) \stackrel{\mathcal{D}}{\longrightarrow}\left(\begin{array}{c}
c \\
W_{m}^{*}
\end{array}\right)
$$

where

$$
W_{m}^{*}(\lambda)=(Z(\lambda)-\lambda Z(1)) \int_{\mathbb{R}} J(x) d F(x), 0 \leq \lambda \leq 1 .
$$

Obviously, the self-normalized Wilcoxon test statistic can be represented as a functional of the random vector $h_{n}$. Hence, an application of the continuous mapping theorem just requires the definition of an appropriate function $G: \bar{M} \times D[0,1] \longrightarrow \mathbb{R}$ that maps $h_{n}$ on $T_{n}\left(\tau_{1}, \tau_{2}\right)=G\left(h_{n}\right)$. For $\lambda \in\left[\tau_{1}, \tau_{2}\right]$ consider the function $G_{\lambda}: \bar{M} \times D[0,1] \longrightarrow \mathbb{R}$ that maps an element $h=\left(h_{1}, h_{2}\right)$ on

$$
\frac{\left|h_{2}(\lambda)\right|}{\left\{\int_{0}^{\lambda}\left(h_{2}(r)-\frac{h_{1}(r)}{h_{1}(\lambda)} h_{2}(\lambda)\right)^{2} d r+\int_{\lambda}^{1}\left(h_{2}(r)-\frac{1-h_{1}(r)}{1-h_{1}(\lambda)} h_{2}(\lambda)\right)^{2} d r\right\}^{\frac{1}{2}}}
$$

provided that the function $F: \bar{M} \times D[0,1] \longrightarrow \mathbb{R}$ defined by

$$
F(h)=\inf _{\lambda \in\left[\tau_{1}, \tau_{2}\right]}\left\{\int_{0}^{\lambda}\left(h_{2}(r)-\frac{h_{1}(r)}{h_{1}(\lambda)} h_{2}(\lambda)\right)^{2} d r+\int_{\lambda}^{1}\left(h_{2}(r)-\frac{1-h_{1}(r)}{1-h_{1}(\lambda)} h_{2}(\lambda)\right)^{2} d r\right\}^{\frac{1}{2}}
$$

does not equal 0 in $h$. Given that $h \in F^{-1}(\{0\})$, we set $G_{\lambda}(h)=-1$.

Since $T_{n}\left(\tau_{1}, \tau_{2}\right)=\sup _{\lambda \in\left[\tau_{1}, \tau_{2}\right]} G_{\lambda}\left(h_{n}\right)$, we intend to apply the continuous mapping theorem to the function $G: \bar{M} \times D[0,1] \longrightarrow \mathbb{R}$, where $G(h)=\sup _{\lambda \in\left[\tau_{1}, \tau_{2}\right]} G_{\lambda}(h)$. Thus, we have to verify that the function $G$ complies with the requirements of the continuous mapping theorem, i.e. we have to prove the following assertions:

1) The function $G$ is measurable with respect to the uniform product metric on $\bar{M} \times D[0,1]$. 
2) We have $P\left(h \in D_{G}\right)=0$, where $D_{G}$ denotes the set of discontinuities of $G$.

In order to show that $G$ is measurable, we consider the restrictions of $G$ to $(\bar{M} \times D[0,1]) \backslash F^{-1}(\{0\})$ and $F^{-1}(\{0\})$, respectively. Both restrictions are continuous with respect to the uniform metric. In particular, both restrictions are Borel measurable. Since the restricted domains are Borel measurable subsets of $\bar{M} \times D[0,1]$, the measurability of the restrictions implies the measurability of $G$.

It remains to show that $P\left(h \in D_{G}\right)=0$. Again, consider the restriction of $G$ to $(\bar{M} \times D[0,1]) \backslash$ $F^{-1}(\{0\})$. Because of the continuity of the restriction, $G$ is continuous at every $h \in(\bar{M} \times D[0,1]) \backslash$ $F^{-1}(\{0\})$ as $F^{-1}(\{0\})$ is a closed subset of $\bar{M} \times D[0,1]$. Therefore, $D_{G}$ is a subset of $F^{-1}(\{0\})$. Consequently, it suffices to show that $P\left(h \in F^{-1}(\{0\})\right)=0$ in order to prove that $P\left(h \in D_{G}\right)=0$.

The random vector $h=\left(c, W_{m}^{*}\right)$ is an element of $F^{-1}(\{0\})$ if and only if the expression

$$
\inf _{\lambda \in\left[\tau_{1}, \tau_{2}\right]}\left\{\int_{0}^{\lambda}\left(f(r)-\frac{r}{\lambda} f(\lambda)\right)^{2} d r+\int_{\lambda}^{1}\left(f(r)-\frac{1-r}{1-\lambda} f(\lambda)\right)^{2} d r\right\}^{\frac{1}{2}}
$$

vanishes when $f=W_{m}^{*}$.

Note that

$$
\begin{aligned}
W_{m}^{*}(r)-\frac{r}{\lambda} W_{m}^{*}(\lambda) & =\int J(x) d F(x)\left\{(Z(r)-r Z(1))-\frac{r}{\lambda}(Z(\lambda)-\lambda Z(1))\right\} \\
& =\int J(x) d F(x)\left\{Z(r)-\frac{r}{\lambda} Z(\lambda)\right\}
\end{aligned}
$$

and

$$
\begin{aligned}
W_{m}^{*}(r)-\frac{1-r}{1-\lambda} W_{m}^{*}(\lambda) & =\int J(x) d F(x)\left\{(Z(r)-r Z(1))-\frac{1-r}{1-\lambda}(Z(\lambda)-\lambda Z(1))\right\} \\
& =\int J(x) d F(x)\left\{Z(r)-Z(\lambda)-\frac{r-\lambda}{1-\lambda}(Z(1)-Z(\lambda))\right\} .
\end{aligned}
$$

Therefore, and as $Z \in C[0,1]$ almost surely (see Maejima and Tudor (2007)), the term in formula (5) vanishes if for some $\lambda \in\left[\tau_{1}, \tau_{2}\right]$

$$
\left\{\int_{0}^{\lambda}\left(V_{m}(r ; 0, \lambda)\right)^{2} d r+\int_{\lambda}^{1}\left(V_{m}(r ; \lambda, 1)\right)^{2} d r\right\}^{\frac{1}{2}}=0
$$

where

$$
V_{m}\left(r ; r_{1}, r_{2}\right)=Z(r)-Z\left(r_{1}\right)-\frac{r-r_{1}}{r_{2}-r_{1}}\left(Z\left(r_{2}\right)-Z\left(r_{1}\right)\right)
$$

It suffices to show that the sample paths of $W_{m}^{*}$ do not belong to the set of continuous functions $f$ that satisfy

$$
\left\{\int_{0}^{\lambda}\left(f(r)-\frac{r}{\lambda} f(\lambda)\right)^{2} d r+\int_{\lambda}^{1}\left(f(r)-f(\lambda)-\frac{r-\lambda}{1-\lambda}(f(1)-f(\lambda))\right)^{2} d r\right\}^{\frac{1}{2}}=0
$$

for some $\lambda \in\left[\tau_{1}, \tau_{2}\right]$. The above equation only holds if the integrands vanish almost surely on the corresponding intervals. In particular, a continuous function $f \in D[0,1]$ that meets formula (8) satisfies

$$
f(r)=\frac{1}{\lambda} f(\lambda) r
$$

if $r \in[0, \lambda]$ and

$$
\begin{aligned}
f(r) & =f(\lambda)+\frac{r-\lambda}{1-\lambda}\{f(1)-f(\lambda)\} \\
& =f(\lambda)-\frac{\lambda}{1-\lambda}\{f(1)-f(\lambda)\}+\frac{1}{1-\lambda}\{f(1)-f(\lambda)\} r
\end{aligned}
$$


if $r \in[\lambda, 1]$. Consequently, the set of continuous functions which lie in $F^{-1}(\{0\})$ corresponds to the class of functions

$$
\begin{aligned}
A= & \left\{f \in D[0,1] \mid \text { for some } \lambda \in\left[\tau_{1}, \tau_{2}\right] \text { and } a, b \in \mathbb{R}\right. \\
& f(r)=\frac{1}{\lambda} \text { ar on }[0, \lambda] \text { and } \\
& \left.f(r)=a-\frac{\lambda}{1-\lambda}\{b-a\}+\frac{1}{1-\lambda}\{b-a\} r \text { on }[\lambda, 1]\right\} .
\end{aligned}
$$

It follows that $P(Z \in A)=0$ because the sample paths of the Hermite process $Z$ are nowhere differentiable with probability 1 (see Mikosch $(1998)$ ), whereas an element in $A$ is differentiable almost everywhere. This implies $P\left(h \in D_{G}\right)=0$.

Having verified the preconditions of the continuous mapping theorem we are now able to conclude that the test statistic $T_{n}\left(\tau_{1}, \tau_{2}\right)$ converges in distribution to

$$
T\left(m, \tau_{1}, \tau_{2}\right)=\sup _{\lambda \in\left[\tau_{1}, \tau_{2}\right]} \frac{\left|W_{m}^{*}(\lambda)\right|}{\left\{\int_{0}^{\lambda}\left(W_{m}^{*}(r)-\frac{r}{\lambda} W_{m}^{*}(\lambda)\right)^{2} d r+\int_{\lambda}^{1}\left(W_{m}^{*}(r)-\frac{1-r}{1-\lambda} W_{m}^{*}(\lambda)\right)^{2} d r\right\}^{\frac{1}{2}}}
$$

Due to (6) and (7), the limit process $T\left(m, \tau_{1}, \tau_{2}\right)$ equals

$$
\sup _{\lambda \in\left[\tau_{1}, \tau_{2}\right]} \frac{|Z(\lambda)-\lambda Z(1)|}{\left\{\int_{0}^{\lambda}\left(V_{m}(r ; 0, \lambda)\right)^{2} d r+\int_{\lambda}^{1}\left(V_{m}(r ; \lambda, 1)\right)^{2} d r\right\}^{\frac{1}{2}}} .
$$

Thus, we have established Theorem 1

In the proof of Theorem 2 we make use of preliminary results stated in Lemma 1, Lemma 2 and Corollary 1. The line of argument that verifies Lemma 1 and Lemma 2 is a modification of the proof that establishes Theorem 3.1 in Dehling, Rooch and Taqqu (2013b).

Lemma 1. Suppose that $\left(\xi_{i}\right)_{i \geq 1}$ is a stationary, long-range dependent Gaussian process with mean 0 , variance 1 and LRD parameter $0<D<\frac{1}{m}$, where $m$ denotes the Hermite rank of the class of functions $1_{\left\{G\left(\xi_{i}\right) \leq x\right\}}-F(x), x \in \mathbb{R}$. Moreover, assume that $\left(G\left(\xi_{i}\right)\right)_{i \geq 1}$ has a continuous distribution function $F$ and that $G: \mathbb{R} \longrightarrow \mathbb{R}$ is a measurable function. Then, if $\Delta \in \mathbb{R}$,

$$
\begin{aligned}
& \frac{1}{n^{2}} \sum_{i=1}^{\lfloor n \lambda\rfloor} \sum_{j=\lfloor n \tau\rfloor+1}^{n} 1_{\left\{G\left(\xi_{i}\right) \leq G\left(\xi_{j}\right)+\Delta\right\}} \stackrel{P}{\longrightarrow} \lambda(1-\tau) \int_{\mathbb{R}} F(x+\Delta) d F(x), \\
& \frac{1}{n^{2}} \sum_{i=1}^{\lfloor n \tau\rfloor} \sum_{j=\lfloor n \lambda\rfloor+1}^{n} 1_{\left\{G\left(\xi_{i}\right) \leq G\left(\xi_{j}\right)+\Delta\right\}} \stackrel{P}{\longrightarrow} \tau(1-\lambda) \int_{\mathbb{R}} F(x+\Delta) d F(x)
\end{aligned}
$$

for fixed $\tau$, uniformly in $\lambda \leq \tau$ and $\lambda \geq \tau$, respectively.

Proof of Lemma 1. We give a proof for the first assertion only as the convergence of the second term follows by an analogous argumentation.

Let $F_{k}$ and $F_{k+1, n}$ denote the empirical distribution functions of the first $k$ and last $n-k$ realizations of $G\left(\xi_{1}\right), \ldots, G\left(\xi_{n}\right)$, i.e.

$$
\begin{aligned}
& F_{k}(x)=\frac{1}{k} \sum_{i=1}^{k} 1_{\left\{G\left(\xi_{i}\right) \leq x\right\}}, \\
& F_{k+1, n}(x)=\frac{1}{n-k} \sum_{i=k+1}^{n} 1_{\left\{G\left(\xi_{i}\right) \leq x\right\}} .
\end{aligned}
$$

For $\lambda \leq \tau$ this yields the following representation:

$$
\sum_{i=1}^{\lfloor n \lambda\rfloor} \sum_{j=\lfloor n \tau\rfloor+1}^{n} 1_{\left\{G\left(\xi_{i}\right) \leq G\left(\xi_{j}\right)+\Delta\right\}}=(n-\lfloor n \tau\rfloor)\lfloor n \lambda\rfloor \frac{1}{n-\lfloor n \tau\rfloor} \sum_{j=\lfloor n \tau\rfloor+1}^{n} F_{\lfloor n \lambda\rfloor}\left(G\left(\xi_{j}\right)+\Delta\right)
$$




$$
=(n-\lfloor n \tau\rfloor)\lfloor n \lambda\rfloor \int_{\mathbb{R}} F_{\lfloor n \lambda\rfloor}(x+\Delta) d F_{\lfloor n \tau\rfloor+1, n}(x)
$$

Since $\frac{n-\lfloor n \tau\rfloor}{n} \longrightarrow 1-\tau$, it suffices to show that $\lfloor n \lambda\rfloor \int_{\mathbb{R}} F_{\lfloor n \lambda\rfloor}(x+\Delta) d F_{\lfloor n \tau\rfloor+1, n}(x)$ converges to $\lambda \int_{\mathbb{R}} F(x+\Delta) d F(x)$. For this purpose, we consider the inequality

$$
\begin{aligned}
& \sup _{0 \leq \lambda \leq \tau}\left|\frac{1}{n}\lfloor n \lambda\rfloor \int_{\mathbb{R}} F_{\lfloor n \lambda\rfloor}(x+\Delta) d F_{\lfloor n \tau\rfloor+1, n}(x)-\lambda \int_{\mathbb{R}} F(x+\Delta) d F(x)\right| \\
& \leq \sup _{0 \leq \lambda \leq \tau}\left|\frac{1}{n} \int_{\mathbb{R}}\lfloor n \lambda\rfloor F_{\lfloor n \lambda\rfloor}(x+\Delta) d F_{\lfloor n \tau\rfloor+1, n}(x)-\frac{\lfloor n \lambda\rfloor}{n} \int_{\mathbb{R}} F(x+\Delta) d F_{\lfloor n \tau\rfloor+1, n}(x)\right| \\
& +\sup _{0 \leq \lambda \leq \tau}\left|\frac{\lfloor n \lambda\rfloor}{n} \int_{\mathbb{R}} F(x+\Delta) d F_{\lfloor n \tau\rfloor+1, n}(x)-\lambda \int_{\mathbb{R}} F(x+\Delta) d F_{\lfloor n \tau\rfloor+1, n}(x)\right| \\
& +\sup _{0 \leq \lambda \leq \tau}\left|\lambda \int_{\mathbb{R}} F(x+\Delta) d F_{\lfloor n \tau\rfloor+1, n}(x)-\lambda \int_{\mathbb{R}} F(x+\Delta) d F(x)\right|
\end{aligned}
$$

and we will show that each of the three terms on its right-hand side converges to 0 .

For the third summand we get

$$
\begin{aligned}
& \sup _{0 \leq \lambda \leq \tau}\left|\lambda \int_{\mathbb{R}} F(x+\Delta) d F_{\lfloor n \tau\rfloor+1, n}(x)-\lambda \int_{\mathbb{R}} F(x+\Delta) d F(x)\right| \\
& =\sup _{0 \leq \lambda \leq \tau}\left|\lambda\left(1-\int_{\mathbb{R}} F_{\lfloor n \tau\rfloor+1, n}(x) d F(x+\Delta)-\left(1-\int_{\mathbb{R}} F(x) d F(x+\Delta)\right)\right)\right| \\
& =\tau\left|\int_{\mathbb{R}}\left(F_{\lfloor n \tau\rfloor+1, n}(x)-F(x)\right) d F(x+\Delta)\right| \\
& \leq \tau \sup _{x \in \mathbb{R}}\left|F_{\lfloor n \tau\rfloor+1, n}(x)-F(x)\right|
\end{aligned}
$$

as a consequence of integration by parts. Furthermore, we have

$$
\sup _{x \in \mathbb{R}}\left|F_{n}(x)-F(x)\right| \longrightarrow 0 \text { a.s. }
$$

by an application of the Glivenko-Cantelli theorem (see Krengel and Brunel (1985)) to the stationary and ergodic process $\left(G\left(\xi_{i}\right)\right)_{i \geq 1}$. So as to deduce an analogous result for $F_{\lfloor n \tau\rfloor+1, n}$ we rewrite

$$
F_{\lfloor n \tau\rfloor+1, n}(x)=\frac{n}{n-\lfloor n \tau\rfloor} F_{n}(x)-\frac{\lfloor n \tau\rfloor}{n-\lfloor n \tau\rfloor} F_{\lfloor n \tau\rfloor}(x)
$$

and we may therefore conclude

$$
\left|F_{\lfloor n \tau\rfloor+1, n}(x)-F(x)\right| \leq\left|\frac{n}{n-\lfloor n \tau\rfloor}\right|\left|F_{n}(x)-F(x)\right|+\left|\frac{\lfloor n \tau\rfloor}{n-\lfloor n \tau\rfloor}\right|\left|F_{\lfloor n \tau\rfloor}(x)-F(x)\right| .
$$

Thus,

$$
\sup _{x \in \mathbb{R}}\left|F_{\lfloor n \tau\rfloor+1, n}(x)-F(x)\right| \longrightarrow 0 \text { a.s. }
$$

which implies that the third term on the right-hand side of (9) converges to 0 almost surely.

Regarding the second term on the right-hand side of (9), we obtain

$$
\begin{aligned}
& \sup _{0 \leq \lambda \leq \tau}\left|\frac{\lfloor n \lambda\rfloor}{n} \int_{\mathbb{R}} F(x+\Delta) d F_{\lfloor n \tau\rfloor+1, n}(x)-\lambda \int_{\mathbb{R}} F(x+\Delta) d F_{\lfloor n \tau\rfloor+1, n}(x)\right| \\
& =\sup _{0 \leq \lambda \leq \tau}\left|\frac{\lfloor n \lambda\rfloor}{n}-\lambda\right|\left|\int_{\mathbb{R}} F(x+\Delta) d F_{\lfloor n \tau\rfloor+1, n}(x)\right| .
\end{aligned}
$$

The right-hand side of this equation converges to 0 since $\left|\int_{\mathbb{R}} F(x+\Delta) d F_{\lfloor n \tau\rfloor+1, n}(x)\right|$ is bounded by 1 , and as

$$
\sup _{0 \leq \lambda \leq \tau}\left|\frac{\lfloor n \lambda\rfloor}{n}-\lambda\right| \longrightarrow 0 .
$$


In order to show that the first term in $(9)$ converges to 0 as well, we consider the following inequality:

$$
\begin{aligned}
& \sup _{0 \leq \lambda \leq \tau}\left|\frac{1}{n} \int_{\mathbb{R}}\lfloor n \lambda\rfloor F_{\lfloor n \lambda\rfloor}(x+\Delta) d F_{\lfloor n \tau\rfloor+1, n}(x)-\frac{\lfloor n \lambda\rfloor}{n} \int_{\mathbb{R}} F(x+\Delta) d F_{\lfloor n \tau\rfloor+1, n}(x)\right| \\
& =\sup _{0 \leq \lambda \leq \tau}\left|\frac{1}{n} \int_{\mathbb{R}}\lfloor n \lambda\rfloor\left(F_{\lfloor n \lambda\rfloor}(x+\Delta)-F(x+\Delta)\right) d F_{\lfloor n \tau\rfloor+1, n}(x)\right| \\
& \leq \sup _{0 \leq \lambda \leq \tau}\left|\frac{d_{n}}{n} \int_{\mathbb{R}} d_{n}^{-1}\lfloor n \lambda\rfloor\left(F_{\lfloor n \lambda\rfloor}(x+\Delta)-F(x+\Delta)\right)-J(x+\Delta) Z(\lambda) d F_{\lfloor n \tau\rfloor+1, n}(x)\right| \\
& +\sup _{0 \leq \lambda \leq \tau}\left|\frac{d_{n}}{n} Z(\lambda) \int_{\mathbb{R}} J(x+\Delta) d F_{\lfloor n \tau\rfloor+1, n}(x)\right|
\end{aligned}
$$

In what follows, we will prove that both terms on the right-hand side of (11) converge to 0 . For this purpose, we make use of the empirical process non-central limit theorem of Dehling and Taqqu (1989) which states that

$$
\left(d_{n}^{-1}\lfloor n \lambda\rfloor\left(F_{\lfloor n \lambda\rfloor}(x)-F(x)\right)\right)_{x \in[-\infty, \infty], \lambda \in[0,1]} \stackrel{\mathcal{D}}{\longrightarrow} J(x) Z(\lambda),
$$

where " $\stackrel{\mathcal{D}}{\longrightarrow}$ " denotes convergence in distribution with respect to the $\sigma$-field generated by the open balls in $D([-\infty, \infty] \times[0,1])$, equipped with the supremum norm.

Due to the Dudley-Wichura version of Skorohod's representation theorem (see Shorack and Wellner (1986), Theorem 2.3.4), we may assume without loss of generality that

$$
\sup _{\lambda, x}\left|d_{n}^{-1}\lfloor n \lambda\rfloor\left(F_{\lfloor n \lambda\rfloor}(x)-F(x)\right)-J(x) Z(\lambda)\right| \longrightarrow 0
$$

almost surely; see Dehling, Rooch and Taqqu (2013a). As a consequence, the first summand in (11) converges to 0 since

$$
\begin{aligned}
& \sup _{0 \leq \lambda \leq \tau}\left|\frac{d_{n}}{n} \int_{\mathbb{R}} d_{n}^{-1}\lfloor n \lambda\rfloor\left(F_{\lfloor n \lambda\rfloor}(x+\Delta)-F(x+\Delta)\right)-J(x+\Delta) Z(\lambda) d F_{\lfloor n \tau\rfloor+1, n}(x)\right| \\
& =\frac{d_{n}}{n} \sup _{0 \leq \lambda \leq \tau, x}\left|d_{n}^{-1}\lfloor n \lambda\rfloor\left(F_{\lfloor n \lambda\rfloor}(x+\Delta)-F(x+\Delta)\right)-J(x+\Delta) Z(\lambda)\right|
\end{aligned}
$$

and as $\frac{d_{n}}{n}$ converges to 0 as well.

For the second summand we get the following inequality:

$$
\sup _{0 \leq \lambda \leq \tau}\left|\frac{d_{n}}{n} Z(\lambda) \int_{\mathbb{R}} J(x+\Delta) d F_{\lfloor n \tau\rfloor+1, n}(x)\right| \leq \frac{d_{n}}{n} \sup _{0 \leq \lambda \leq \tau}|Z(\lambda)|\left|\int_{\mathbb{R}} J(x+\Delta) d F_{\lfloor n \tau\rfloor+1, n}(x)\right|
$$

Note that

$$
\begin{aligned}
J(x) & =\int_{\mathbb{R}} 1_{\{G(y) \leq x\}} H_{m}(y) \varphi(y) d y \\
& =\int_{\mathbb{R}} H_{m}(y) \varphi(y) d y-\int_{\mathbb{R}} 1_{\{x \leq G(y)\}} H_{m}(y) \varphi(y) d y \\
& =-\int_{\mathbb{R}} 1_{\{x \leq G(y)\}} H_{m}(y) \varphi(y) d y,
\end{aligned}
$$

where $\varphi$ denotes the standard normal density function, since

$$
\int_{\mathbb{R}} H_{m}(y) \varphi(y) d y=0 .
$$

For this reason, we have

$$
\begin{aligned}
\int_{\mathbb{R}} J(x+\Delta) d F_{\lfloor n \tau\rfloor+1, n}(x) & =-\int_{\mathbb{R}} \int_{\mathbb{R}} 1_{\{x+\Delta \leq G(y)\}} H_{m}(y) \varphi(y) d y d F_{\lfloor n \tau\rfloor+1, n}(x) \\
& =-\int_{\mathbb{R}} \int_{\mathbb{R}} 1_{\{x+\Delta \leq G(y)\}} d F_{\lfloor n \tau\rfloor+1, n}(x) H_{m}(y) \varphi(y) d y
\end{aligned}
$$




$$
=-\int_{\mathbb{R}} F_{\lfloor n \tau\rfloor+1, n}(G(y)-\Delta) H_{m}(y) \varphi(y) d y
$$

and

$$
\begin{aligned}
\int_{\mathbb{R}} J(x+\Delta) d F(x) & =-\int_{\mathbb{R}} \int_{\mathbb{R}} 1_{\{x+\Delta \leq G(y)\}} H_{m}(y) \varphi(y) d y d F(x) \\
& =-\int_{\mathbb{R}} \int_{\mathbb{R}} 1_{\{x+\Delta \leq G(y)\}} d F(x) H_{m}(y) \varphi(y) d y \\
& =-\int_{\mathbb{R}} F(G(y)-\Delta) H_{m}(y) \varphi(y) d y .
\end{aligned}
$$

Regarding the difference of these terms, we obtain

$$
\begin{aligned}
& \left|\int_{\mathbb{R}} J(x+\Delta) d F_{\lfloor n \tau\rfloor+1, n}(x)-\int_{\mathbb{R}} J(x+\Delta) d F(x)\right| \\
& =\left|\int_{\mathbb{R}}\left(F(G(y)-\Delta)-F_{\lfloor n \tau\rfloor+1, n}(G(y)-\Delta)\right) H_{m}(y) \varphi(y) d y\right| \\
& \leq \int_{\mathbb{R}}\left|F(G(y)-\Delta)-F_{\lfloor n \tau\rfloor+1, n}(G(y)-\Delta)\right|\left|H_{m}(y)\right| \varphi(y) d y \\
& \leq \sup _{y \in \mathbb{R}}\left|F(G(y)-\Delta)-F_{\lfloor n \tau\rfloor+1, n}(G(y)-\Delta)\right| \int_{\mathbb{R}}\left|H_{m}(y)\right| \varphi(y) d y,
\end{aligned}
$$

where $\int_{\mathbb{R}}\left|H_{m}(y)\right| \varphi(y) d y<\infty$ because of Hölder's inequality and where

$$
\sup _{y \in \mathbb{R}}\left|F(G(y)-\Delta)-F_{\lfloor n \tau\rfloor+1, n}(G(y)-\Delta)\right| \longrightarrow 0 \text { a.s. }
$$

by (10). As a result, $\int_{\mathbb{R}} J(x+\Delta) d F_{\lfloor n \tau\rfloor+1, n}(x) \stackrel{\mathcal{D}}{\longrightarrow} \int_{\mathbb{R}} J(x+\Delta) d F(x)$, so that in the end the second summand in (11) converges to 0 almost surely, too.

All in all, the third term on the right-hand side of (9) converges to 0 almost surely as it is dominated by the sum of two expressions which both converge to 0 with probability 1 . This completes the proof of the first assertion in Lemma 1 .

Corollary 1. Suppose that $\left(\xi_{i}\right)_{i>1}$ is a stationary, long-range dependent Gaussian process with mean 0, variance 1 and LRD parameter $0<D<\frac{1}{m}$, where $m$ denotes the Hermite rank of the class of functions $1_{\left\{G\left(\xi_{i}\right) \leq x\right\}}-F(x), x \in \mathbb{R}$. Moreover, assume that $\left(G\left(\xi_{i}\right)\right)_{i \geq 1}$ has a continuous distribution function $F$ and that $G: \mathbb{R} \longrightarrow \mathbb{R}$ is a measurable function. Then

$$
\frac{1}{n^{2}} \sum_{i=1}^{\lfloor n \tau\rfloor} \sum_{j=\lfloor n \tau\rfloor+1}^{n} 1_{\left\{G\left(\xi_{i}\right) \leq G\left(\xi_{j}\right)+\Delta\right\}} \stackrel{P}{\longrightarrow} \tau(1-\tau) \int_{\mathbb{R}} F(x+\Delta) d F(x)
$$

for fixed $\tau$.

Proof of Corollary 1 . Consider the function $G: D[0, \tau] \longrightarrow \mathbb{R}, f \mapsto f(\tau)$. As $G$ is continuous with respect to the supremum norm on $D[0, \tau]$, Corollary 1 follows from Lemma 1 and the continuous mapping theorem

Lemma 2. Suppose that $\left(\xi_{i}\right)_{i \geq 1}$ is a stationary, long-range dependent Gaussian process with mean 0, variance 1 and LRD parameter $0<D<\frac{1}{m}$, where $m$ denotes the Hermite rank of the class of functions $1_{\left\{G\left(\xi_{i}\right) \leq x\right\}}-F(x), x \in \mathbb{R}$. Moreover, assume that $\left(G\left(\xi_{i}\right)\right)_{i \geq 1}$ has a continuous distribution function $F$ and that $G: \mathbb{R} \longrightarrow \mathbb{R}$ is a measurable function. Then

$$
\frac{1}{n^{2}} \sum_{i=1}^{\lfloor n \tau\rfloor} \sum_{j=\lfloor n \tau\rfloor+1}^{\lfloor n \lambda\rfloor} 1_{\left\{G\left(\xi_{i}\right) \leq G\left(\xi_{j}\right)+\Delta\right\}} \stackrel{P}{\longrightarrow} \tau(\lambda-\tau) \int_{\mathbb{R}} F(x+\Delta) d F(x)
$$

for fixed $\tau$, uniformly in $\lambda \geq \tau$. 
Proof of Lemma 2. Let $F_{k+1, t}$ denote the empirical distribution function of $G\left(\xi_{k+1}\right), \ldots, G\left(\xi_{t}\right)$, i.e.

$$
F_{k+1, t}(x)=\frac{1}{t-k} \sum_{i=k+1}^{t} 1_{\left\{G\left(\xi_{i}\right) \leq x\right\}}
$$

We may therefore rewrite

$$
\begin{aligned}
\sum_{i=1}^{\lfloor n \tau\rfloor} \sum_{j=\lfloor n \tau\rfloor+1}^{\lfloor n \lambda\rfloor} 1_{\left\{G\left(\xi_{i}\right) \leq G\left(\xi_{j}\right)+\Delta\right\}} & =(\lfloor n \lambda\rfloor-\lfloor n \tau\rfloor)\lfloor n \tau\rfloor \frac{1}{(\lfloor n \lambda\rfloor-\lfloor n \tau\rfloor)} \sum_{j=\lfloor n \tau\rfloor+1}^{\lfloor n \lambda\rfloor} F_{\lfloor n \tau\rfloor}\left(G\left(\xi_{j}\right)+\Delta\right) \\
& =(\lfloor n \lambda\rfloor-\lfloor n \tau\rfloor)\lfloor n \tau\rfloor \int_{\mathbb{R}} F_{\lfloor n \tau\rfloor}(x+\Delta) d F_{\lfloor n \tau\rfloor+1,\lfloor n \lambda\rfloor}(x) .
\end{aligned}
$$

Furthermore, repeated application of the triangle inequality yields

$$
\begin{aligned}
& \sup _{\tau \leq \lambda \leq 1}\left|\frac{1}{n}(\lfloor n \lambda\rfloor-\lfloor n \tau\rfloor) \int_{\mathbb{R}} F_{\lfloor n \tau\rfloor}(x+\Delta) d F_{\lfloor n \tau\rfloor+1,\lfloor n \lambda\rfloor}(x)-(\lambda-\tau) \int_{\mathbb{R}} F(x+\Delta) d F(x)\right| \\
& \leq \sup _{\tau \leq \lambda \leq 1}\left|\frac{1}{n}(\lfloor n \lambda\rfloor-\lfloor n \tau\rfloor) \int_{\mathbb{R}}\left(F_{\lfloor n \tau\rfloor}(x+\Delta)-F(x+\Delta)\right) d F_{\lfloor n \tau\rfloor+1,\lfloor n \lambda\rfloor}(x)\right| \\
& \quad+\sup _{\tau \leq \lambda \leq 1}\left|\frac{1}{n}(\lfloor n \lambda\rfloor-\lfloor n \tau\rfloor) \int_{\mathbb{R}} F(x+\Delta) d F_{\lfloor n \tau\rfloor+1,\lfloor n \lambda\rfloor}(x)-\frac{1}{n}(\lfloor n \lambda\rfloor-\lfloor n \tau\rfloor) \int_{\mathbb{R}} F(x+\Delta) d F(x)\right| \\
& \quad+\sup _{\tau \leq \lambda \leq 1}\left|\frac{1}{n}(\lfloor n \lambda\rfloor-\lfloor n \tau\rfloor) \int_{\mathbb{R}} F(x+\Delta) d F(x)-(\lambda-\tau) \int_{\mathbb{R}} F(x+\Delta) d F(x)\right| .
\end{aligned}
$$

In order to prove that the stochastic process considered in Lemma 2 converges to the given limit process, it is sufficient to show that the expressions on the right-hand side of the above inequality converge to 0 . We consider each of the three summands separately.

Apparently, the third term converges to 0 since

$$
\begin{aligned}
& \sup _{\tau \leq \lambda \leq 1}\left|\frac{1}{n}(\lfloor n \lambda\rfloor-\lfloor n \tau\rfloor) \int_{\mathbb{R}} F(x+\Delta) d F(x)-(\lambda-\tau) \int_{\mathbb{R}} F(x+\Delta) d F(x)\right| \\
& =\sup _{\tau \leq \lambda \leq 1}\left|\frac{1}{n}(\lfloor n \lambda\rfloor-\lfloor n \tau\rfloor)-(\lambda-\tau)\right| \int_{\mathbb{R}} F(x+\Delta) d F(x)
\end{aligned}
$$

and $\operatorname{asup}_{\tau \leq \lambda \leq 1}\left|\frac{1}{n}(\lfloor n \lambda\rfloor-\lfloor n \tau\rfloor)-(\lambda-\tau)\right| \longrightarrow 0$.

We have

$$
\begin{aligned}
& \sup _{\tau \leq \lambda \leq 1}\left|\frac{1}{n}(\lfloor n \lambda\rfloor-\lfloor n \tau\rfloor) \int_{\mathbb{R}}\left(F_{\lfloor n \tau\rfloor}(x+\Delta)-F(x+\Delta)\right) d F_{\lfloor n \tau\rfloor+1,\lfloor n \lambda\rfloor}(x)\right| \\
& \leq \sup _{x \in \mathbb{R}}\left|F_{\lfloor n \tau\rfloor}(x+\Delta)-F(x+\Delta)\right| \sup _{\tau \leq \lambda \leq 1}\left|\frac{1}{n}(\lfloor n \lambda\rfloor-\lfloor n \tau\rfloor)\right|
\end{aligned}
$$

for the first summand. As $\sup _{x \in \mathbb{R}}\left|F_{\lfloor n \tau\rfloor}(x+\Delta)-F(x+\Delta)\right|$ converges to 0 almost surely by the Glivenko-Cantelli theorem, so does the right-hand side of the above inequality.

Finally, consider the second term on the right-hand side of $(12)$. We have

$$
\begin{aligned}
& \sup _{\tau \leq \lambda \leq 1}\left|\frac{1}{n}(\lfloor n \lambda\rfloor-\lfloor n \tau\rfloor) \int_{\mathbb{R}} F(x+\Delta) d F_{\lfloor n \tau\rfloor+1,\lfloor n \lambda\rfloor}(x)-\frac{1}{n}(\lfloor n \lambda\rfloor-\lfloor n \tau\rfloor) \int_{\mathbb{R}} F(x+\Delta) d F(x)\right| \\
& =\sup _{\tau \leq \lambda \leq 1}\left|\frac{1}{n}(\lfloor n \lambda\rfloor-\lfloor n \tau\rfloor) \int_{\mathbb{R}} F(x+\Delta) d\left(F_{\lfloor n \tau\rfloor+1,\lfloor n \lambda\rfloor}-F\right)(x)\right| \\
& =\sup _{\tau \leq \lambda \leq 1}\left|\frac{1}{n}(\lfloor n \lambda\rfloor-\lfloor n \tau\rfloor) \int_{\mathbb{R}}\left(F_{\lfloor n \tau\rfloor+1,\lfloor n \lambda\rfloor}(x)-F(x)\right) d F(x+\Delta)\right| \\
& =\frac{d_{n}}{n} \sup _{\tau \leq \lambda \leq 1}\left|\int_{\mathbb{R}} d_{n}^{-1}(\lfloor n \lambda\rfloor-\lfloor n \tau\rfloor)\left(F_{\lfloor n \tau\rfloor+1,\lfloor n \lambda\rfloor}(x)-F(x)\right)-J(x)(Z(\lambda)-Z(\tau)) d F(x+\Delta)\right| \\
& \quad+\sup _{\tau \leq \lambda \leq 1}\left|\frac{d_{n}}{n}(Z(\lambda)-Z(\tau)) \int_{\mathbb{R}} J(x) d F(x+\Delta)\right|
\end{aligned}
$$




$$
\begin{aligned}
\leq & \frac{d_{n}}{n} \sup _{\tau \leq \lambda \leq 1, x \in \mathbb{R}}\left|d_{n}^{-1}(\lfloor n \lambda\rfloor-\lfloor n \tau\rfloor)\left(F_{\lfloor n \tau\rfloor+1,\lfloor n \lambda\rfloor}(x)-F(x)\right)-J(x)(Z(\lambda)-Z(\tau))\right| \\
& +\frac{d_{n}}{n} \sup _{\tau \leq \lambda \leq 1}|Z(\lambda)-Z(\tau)|\left|\int_{\mathbb{R}} J(x) d F(x+\Delta)\right| .
\end{aligned}
$$

It follows from integration by parts that

$$
\begin{aligned}
& \int_{\mathbb{R}} F(x+\Delta) d F_{\lfloor n \tau\rfloor+1,\lfloor n \lambda\rfloor}(x)-\int_{\mathbb{R}} F(x+\Delta) d F(x) \\
& =\int_{\mathbb{R}} F(x) d F(x+\Delta)-\int_{\mathbb{R}} F_{\lfloor n \tau\rfloor+1,\lfloor n \lambda\rfloor}(x) d F(x+\Delta) .
\end{aligned}
$$

Furthermore,

$$
\begin{aligned}
(\lfloor n \lambda\rfloor-\lfloor n \tau\rfloor)\left(F_{\lfloor n \tau\rfloor+1,\lfloor n \lambda\rfloor}(x)-F(x)\right) & =\sum_{i=\lfloor n \tau\rfloor+1}^{\lfloor n \lambda\rfloor} 1_{\left\{G\left(\xi_{i}\right) \leq x\right\}}-(\lfloor n \lambda\rfloor-\lfloor n \tau\rfloor) F(x) \\
& =\lfloor n \lambda\rfloor F_{\lfloor n \lambda\rfloor}(x)-\lfloor n \tau\rfloor F_{\lfloor n \tau\rfloor}(x)-\lfloor n \lambda\rfloor F(x)+\lfloor n \tau\rfloor F(x) \\
& =\lfloor n \lambda\rfloor\left(F_{\lfloor n \lambda\rfloor}(x)-F(x)\right)-\lfloor n \tau\rfloor\left(F_{\lfloor n \tau\rfloor}(x)-F(x)\right) .
\end{aligned}
$$

As a result,

$$
\begin{aligned}
& \sup _{\tau \leq \lambda \leq 1, x \in \mathbb{R}}\left|d_{n}^{-1}(\lfloor n \lambda\rfloor-\lfloor n \tau\rfloor)\left(F_{\lfloor n \tau\rfloor+1,\lfloor n \lambda\rfloor}(x)-F(x)\right)-J(x)(Z(\lambda)-Z(\tau))\right| \\
& =\sup _{\tau \leq \lambda \leq 1, x \in \mathbb{R}}\left|d_{n}^{-1}\left(\lfloor n \lambda\rfloor\left(F_{\lfloor n \lambda\rfloor}(x)-F(x)\right)-\lfloor n \tau\rfloor\left(F_{\lfloor n \tau\rfloor}(x)-F(x)\right)\right)-J(x)(Z(\lambda)-Z(\tau))\right| \\
& \leq \sup _{\tau \leq \lambda \leq 1, x \in \mathbb{R}}\left|d_{n}^{-1}\lfloor n \lambda\rfloor\left(F_{\lfloor n \lambda\rfloor}(x)-F(x)\right)-J(x) Z(\lambda)\right| \\
& \quad+\sup _{\tau \leq \lambda \leq 1, x \in \mathbb{R}}\left|d_{n}^{-1}\lfloor n \tau\rfloor\left(F_{\lfloor n \tau\rfloor}(x)-F(x)\right)-J(x) Z(\tau)\right| .
\end{aligned}
$$

Again, we may assume without loss of generality that

$$
\sup _{\lambda, x}\left|d_{n}^{-1}\lfloor n \lambda\rfloor\left(F_{\lfloor n \lambda\rfloor}(x)-F(x)\right)-J(x) Z(\lambda)\right| \longrightarrow 0
$$

almost surely, as pointed out in the proof of Lemma 1. Since $\frac{d_{n}}{n} \longrightarrow 0$ by definition of $d_{n}$, we may conclude that the third summand on the right hand side of 12 converges to 0 , too. This completes the proof of Lemma 2 .

Proof of Theorem 2, We have

$$
\begin{aligned}
T_{n}\left(\tau_{1}, \tau_{2}\right) & =\sup _{k \in\left[\left\lfloor n \tau_{1}\right\rfloor,\left\lfloor n \tau_{2}\right\rfloor\right]} G_{n}(k) \\
& \geq G_{n}\left(k^{*}\right),
\end{aligned}
$$

where

$$
G_{n}\left(k^{*}\right)=\frac{\left|\sum_{i=1}^{k^{*}} \sum_{j=k^{*}+1}^{n}\left(1_{\left\{X_{i} \leq X_{j}\right\}}-\frac{1}{2}\right)\right|}{\left\{\frac{1}{n} \sum_{t=1}^{k^{*}} S_{t}^{2}\left(1, k^{*}\right)+\frac{1}{n} \sum_{t=k^{*}+1}^{n} S_{t}^{2}\left(k^{*}+1, n\right)\right\}^{\frac{1}{2}}}
$$

and where $k^{*}=\lfloor n \tau\rfloor$ denotes the location of the change-point. Thus, it suffices to show that $G_{n}\left(k^{*}\right) \stackrel{P}{\longrightarrow}$ $\infty$. For this purpose, we rewrite

$$
G_{n}\left(k^{*}\right)=\frac{\frac{1}{n^{2}}\left|\sum_{i=1}^{k^{*}} \sum_{j=k^{*}+1}^{n}\left(1_{\left\{X_{i} \leq X_{j}\right\}}-\frac{1}{2}\right)\right|}{\frac{1}{n^{2}}\left\{\frac{1}{n} \sum_{t=1}^{k^{*}} S_{t}^{2}\left(1, k^{*}\right)+\frac{1}{n} \sum_{t=k^{*}+1}^{n} S_{t}^{2}\left(k^{*}+1, n\right)\right\}^{\frac{1}{2}}} .
$$


We will prove that the numerator of $G_{n}\left(k^{*}\right)$ converges to a positive constant, whereas the denominator tends to 0 in order to show divergence to $\infty$.

First, we turn to the denominator, which equals

$$
\frac{1}{n^{2}}\left\{\int_{0}^{\tau} S_{\lfloor n r\rfloor}^{2}\left(1, k^{*}\right) d r+\int_{\tau}^{1} S_{\lfloor n r\rfloor}^{2}\left(k^{*}+1, n\right) d r\right\}^{\frac{1}{2}} .
$$

Note that for $i \leq k^{*}$

$$
\begin{aligned}
\sum_{j=1}^{n} 1_{\left\{X_{i} \leq X_{j}\right\}} & =\sum_{j=1}^{k^{*}} 1_{\left\{\mu+G\left(\xi_{i}\right) \leq \mu+G\left(\xi_{j}\right)\right\}}+\sum_{j=k^{*}+1}^{n} 1_{\left\{\mu+G\left(\xi_{i}\right) \leq \mu+G\left(\xi_{j}\right)+\Delta\right\}} \\
& =\sum_{j=1}^{n} 1_{\left\{G\left(\xi_{i}\right) \leq G\left(\xi_{j}\right)\right\}}+\sum_{j=k^{*}+1}^{n} 1_{\left\{G\left(\xi_{j}\right)<G\left(\xi_{i}\right) \leq G\left(\xi_{j}\right)+\Delta\right\}} .
\end{aligned}
$$

Therefore,

$$
\begin{aligned}
S_{t}\left(1, k^{*}\right)= & -\sum_{h=1}^{t}\left(\sum_{j=1}^{n} 1_{\left\{X_{h} \leq X_{j}\right\}}-\frac{1}{k^{*}} \sum_{i=1}^{k^{*}} \sum_{j=1}^{n} 1_{\left\{X_{i} \leq X_{j}\right\}}\right) \\
= & -\sum_{h=1}^{t}\left(\sum_{j=1}^{n} 1_{\left\{G\left(\xi_{h}\right) \leq G\left(\xi_{j}\right)\right\}}-\frac{1}{k^{*}} \sum_{i=1}^{k^{*}} \sum_{j=1}^{n} 1_{\left\{G\left(\xi_{i}\right) \leq G\left(\xi_{j}\right)\right\}}\right) \\
& -\sum_{h=1}^{t}\left(\sum_{j=k^{*}+1}^{n} 1_{\left\{G\left(\xi_{j}\right)<G\left(\xi_{h}\right) \leq G\left(\xi_{j}\right)+\Delta\right\}}-\frac{1}{k^{*}} \sum_{i=1}^{k^{*}} \sum_{j=k^{*}+1}^{n} 1_{\left\{G\left(\xi_{j}\right)<G\left(\xi_{i}\right) \leq G\left(\xi_{j}\right)+\Delta\right\}}\right) .
\end{aligned}
$$

We treat the expression $S_{t}\left(1, k^{*}\right)$ as sum of the following terms

$$
\begin{aligned}
& \hat{S}_{t}\left(1, k^{*}\right)=-\sum_{h=1}^{t}\left(\sum_{j=1}^{n} 1_{\left\{G\left(\xi_{h}\right) \leq G\left(\xi_{j}\right)\right\}}-\frac{1}{k^{*}} \sum_{i=1}^{k^{*}} \sum_{j=1}^{n} 1_{\left\{G\left(\xi_{i}\right) \leq G\left(\xi_{j}\right)\right\}}\right) \\
& \tilde{S}_{t}\left(1, k^{*}\right)=-\sum_{h=1}^{t}\left(\sum_{j=k^{*}+1}^{n} 1_{\left\{G\left(\xi_{j}\right)<G\left(\xi_{h}\right) \leq G\left(\xi_{j}\right)+\Delta\right\}}-\frac{1}{k^{*}} \sum_{i=1}^{k^{*}} \sum_{j=k^{*}+1}^{n} 1_{\left\{G\left(\xi_{j}\right)<G\left(\xi_{i}\right) \leq G\left(\xi_{j}\right)+\Delta\right\}}\right) .
\end{aligned}
$$

For the first summand we get

$$
\begin{aligned}
\hat{S}_{t}\left(1, k^{*}\right)= & -\sum_{h=1}^{t}\left(\sum_{j=1}^{n} 1_{\left\{G\left(\xi_{h}\right) \leq G\left(\xi_{j}\right)\right\}}-\frac{1}{k^{*}} \sum_{i=1}^{k^{*}} \sum_{j=1}^{n} 1_{\left\{G\left(\xi_{i}\right) \leq G\left(\xi_{j}\right)\right\}}\right) \\
= & -\sum_{i=1}^{t} \sum_{j=1}^{t} 1_{\left\{G\left(\xi_{i}\right) \leq G\left(\xi_{j}\right)\right\}}-\sum_{i=1}^{t} \sum_{j=t+1}^{n} 1_{\left\{G\left(\xi_{i}\right) \leq G\left(\xi_{j}\right)\right\}} \\
& +\frac{t}{k^{*}} \sum_{i=1}^{k^{*}} \sum_{j=1}^{k^{*}} 1_{\left\{G\left(\xi_{i}\right) \leq G\left(\xi_{j}\right)\right\}}+\frac{t}{k^{*}} \sum_{i=1}^{k^{*}} \sum_{j=k^{*}+1}^{n} 1_{\left\{G\left(\xi_{i}\right) \leq G\left(\xi_{j}\right)\right\}} \\
= & -\frac{t(t+1)}{2}-\sum_{i=1}^{t} \sum_{j=t+1}^{n} 1_{\left\{G\left(\xi_{i}\right) \leq G\left(\xi_{j}\right)\right\}}+\frac{t}{k^{*}} \frac{k^{*}\left(k^{*}+1\right)}{2}+\frac{t}{k^{*}} \sum_{i=1}^{k^{*}} \sum_{j=k^{*}+1}^{n} 1_{\left\{G\left(\xi_{i}\right) \leq G\left(\xi_{j}\right)\right\}} \\
= & -\frac{t^{2}}{2}-\sum_{i=1}^{t} \sum_{j=t+1}^{n} 1_{\left\{G\left(\xi_{i}\right) \leq G\left(\xi_{j}\right)\right\}}+\frac{t k^{*}}{2}+\frac{t}{k^{*}} \sum_{i=1}^{k^{*}} \sum_{j=k^{*}+1}^{n} 1_{\left\{G\left(\xi_{i}\right) \leq G\left(\xi_{j}\right)\right\} .} .
\end{aligned}
$$

We have

$$
\frac{1}{n^{2}} \sum_{i=1}^{\lfloor n \lambda\rfloor} \sum_{j=\lfloor n \lambda\rfloor+1}^{n} 1_{\left\{G\left(\xi_{i}\right) \leq G\left(\xi_{j}\right)\right\}} \stackrel{P}{\longrightarrow} \frac{\lambda(1-\lambda)}{2}
$$


uniformly in $\lambda$ because

$$
\frac{1}{n d_{n}} \sum_{i=1}^{\lfloor n \lambda\rfloor} \sum_{j=\lfloor n \lambda\rfloor+1}^{n}\left(1_{\left\{G\left(\xi_{i}\right) \leq G\left(\xi_{j}\right)\right\}}-\frac{1}{2}\right) \stackrel{\mathcal{D}}{\longrightarrow}(Z(\lambda)-\lambda Z(1)) \int_{\mathbb{R}} J(x) d F(x)
$$

uniformly in $\lambda$ by Theorem 1.1 in Dehling, Rooch and Taqqu (2013a) and as $\frac{d_{n}}{n} \longrightarrow 0$. We may conclude from this and Corollary 1 that $\frac{1}{n^{2}} \hat{S}_{\lfloor n \lambda\rfloor}\left(1,\left\lfloor k^{*}\right\rfloor\right) \stackrel{P}{\longrightarrow} 0$ uniformly in $\lambda \leq \tau$.

Because of

$$
1_{\left\{G\left(\xi_{j}\right)<G\left(\xi_{i}\right) \leq G\left(\xi_{j}\right)+\Delta\right\}}=1_{\left\{G\left(\xi_{i}\right) \leq G\left(\xi_{j}\right)+\Delta\right\}}-1_{\left\{G\left(\xi_{i}\right) \leq G\left(\xi_{j}\right)\right\}},
$$

the second summand can be written as

$$
\begin{aligned}
\tilde{S}_{t}\left(1, k^{*}\right)= & -\sum_{h=1}^{t}\left(\sum_{j=k^{*}+1}^{n} 1_{\left\{G\left(\xi_{j}\right)<G\left(\xi_{h}\right) \leq G\left(\xi_{j}\right)+\Delta\right\}}-\frac{1}{k^{*}} \sum_{i=1}^{k^{*}} \sum_{j=k^{*}+1}^{n} 1_{\left\{G\left(\xi_{j}\right)<G\left(\xi_{i}\right) \leq G\left(\xi_{j}\right)+\Delta\right\}}\right) \\
= & -\sum_{i=1}^{t} \sum_{j=k^{*}+1}^{n} 1_{\left\{G\left(\xi_{i}\right) \leq G\left(\xi_{j}\right)+\Delta\right\}}+\frac{t}{k^{*}} \sum_{i=1}^{k^{*}} \sum_{j=k^{*}+1}^{n} 1_{\left\{G\left(\xi_{i}\right) \leq G\left(\xi_{j}\right)+\Delta\right\}} \\
& +\sum_{i=1}^{t} \sum_{j=k^{*}+1}^{n} 1_{\left\{G\left(\xi_{i}\right) \leq G\left(\xi_{j}\right)\right\}}-\frac{t}{k^{*}} \sum_{i=1}^{k^{*}} \sum_{j=k^{*}+1}^{n} 1_{\left\{G\left(\xi_{i}\right) \leq G\left(\xi_{j}\right)\right\}} .
\end{aligned}
$$

Due to Lemma 1 and Corollary $1, \frac{1}{n^{2}} \tilde{S}_{\lfloor n \lambda\rfloor}\left(1, k^{*}\right)$ converges in probability to 0 , as well.

All in all, the previous considerations yield

$$
\int_{0}^{\tau}\left\{\frac{1}{n^{2}} S_{\lfloor n r\rfloor}\left(1, k^{*}\right)\right\}^{2} d r \stackrel{P}{\longrightarrow} 0
$$

as $G: D[0, \tau] \longrightarrow \mathbb{R}, f \mapsto \int_{0}^{\tau}(f(s))^{2} d s$, is continuous with respect to the supremum norm on $D[0, \tau]$. In analogy to the previous argumentation it can be shown that

$$
\int_{\tau}^{1}\left\{\frac{1}{n^{2}} S_{\lfloor n r\rfloor}\left(k^{*}+1, n\right)\right\}^{2} d r \stackrel{P}{\longrightarrow} 0 .
$$

For this purpose, note that, if $i>k^{*}$,

$$
\begin{aligned}
\sum_{j=1}^{n} 1_{\left\{X_{i} \leq X_{j}\right\}} & =\sum_{j=1}^{k^{*}} 1_{\left\{\mu+G\left(\xi_{i}\right)+\Delta \leq \mu+G\left(\xi_{j}\right)\right\}}+\sum_{j=k^{*}+1}^{n} 1_{\left\{\mu+G\left(\xi_{i}\right)+\Delta \leq \mu+G\left(\xi_{j}\right)+\Delta\right\}} \\
& =\sum_{j=1}^{n} 1_{\left\{G\left(\xi_{i}\right) \leq G\left(\xi_{j}\right)\right\}}-\sum_{j=1}^{k^{*}} 1_{\left\{G\left(\xi_{i}\right) \leq G\left(\xi_{j}\right)<G\left(\xi_{i}\right)+\Delta\right\}} .
\end{aligned}
$$

Therefore,

$$
\begin{aligned}
S_{t}\left(k^{*}+1, n\right)= & -\sum_{h=k^{*}+1}^{t}\left(\sum_{j=1}^{n} 1_{\left\{X_{h} \leq X_{j}\right\}}-\frac{1}{n-k^{*}} \sum_{i=k^{*}+1}^{n} \sum_{j=1}^{n} 1_{\left\{X_{i} \leq X_{j}\right\}}\right) \\
= & -\sum_{h=k^{*}+1}^{t}\left(\sum_{j=1}^{n} 1_{\left\{G\left(\xi_{h}\right) \leq G\left(\xi_{j}\right)\right\}}-\frac{1}{n-k^{*}} \sum_{i=k^{*}+1}^{n} \sum_{j=1}^{n} 1_{\left\{G\left(\xi_{i}\right) \leq G\left(\xi_{j}\right)\right\}}\right) \\
& +\sum_{h=k^{*}+1}^{t}\left(\sum_{j=1}^{k^{*}} 1_{\left\{G\left(\xi_{h}\right) \leq G\left(\xi_{j}\right)<G\left(\xi_{h}\right)+\Delta\right\}}-\frac{1}{n-k^{*}} \sum_{i=k^{*}+1}^{n} \sum_{j=1}^{k^{*}} 1_{\left\{G\left(\xi_{i}\right) \leq G\left(\xi_{j}\right)<G\left(\xi_{i}\right)+\Delta\right\}}\right)
\end{aligned}
$$

Hence, we consider $S_{t}\left(k^{*}+1, n\right)$ as sum of the expressions below

$$
\hat{S}_{t}\left(k^{*}+1, n\right)=-\sum_{h=k^{*}+1}^{t}\left(\sum_{j=1}^{n} 1_{\left\{G\left(\xi_{h}\right) \leq G\left(\xi_{j}\right)\right\}}-\frac{1}{n-k^{*}} \sum_{i=k^{*}+1}^{n} \sum_{j=1}^{n} 1_{\left\{G\left(\xi_{i}\right) \leq G\left(\xi_{j}\right)\right\}}\right),
$$




$$
\tilde{S}_{t}\left(k^{*}+1, n\right)=\sum_{h=k^{*}+1}^{t}\left(\sum_{j=1}^{k^{*}} 1_{\left\{G\left(\xi_{h}\right) \leq G\left(\xi_{j}\right)<G\left(\xi_{h}\right)+\Delta\right\}}-\frac{1}{n-k^{*}} \sum_{i=k^{*}+1}^{n} \sum_{j=1}^{k^{*}} 1_{\left\{G\left(\xi_{i}\right) \leq G\left(\xi_{j}\right)<G\left(\xi_{i}\right)+\Delta\right\}}\right) .
$$

The following representation arises from rather simple transformations

$$
\begin{aligned}
\hat{S}_{t}\left(k^{*}+1, n\right)= & -\sum_{h=k^{*}+1}^{t}\left(\sum_{j=1}^{n} 1_{\left\{G\left(\xi_{n}\right) \leq G\left(\xi_{j}\right)\right\}}-\frac{1}{n-k^{*}} \sum_{i=k^{*}+1}^{n} \sum_{j=1}^{n} 1_{\left\{G\left(\xi_{i}\right) \leq G\left(\xi_{j}\right)\right\}}\right) \\
= & -\sum_{i=1}^{t} \sum_{j=t+1}^{n} 1_{\left\{G\left(\xi_{i}\right) \leq G\left(\xi_{j}\right)\right\}}-\sum_{i=1}^{t} \sum_{j=1}^{t} 1_{\left\{G\left(\xi_{i}\right) \leq G\left(\xi_{j}\right)\right\}} \\
& +\sum_{i=1}^{k^{*}} \sum_{j=k^{*}+1}^{n} 1_{\left\{G\left(\xi_{i}\right) \leq G\left(\xi_{j}\right)\right\}}+\sum_{i=1}^{k^{*}} \sum_{j=1}^{k^{*}} 1_{\left\{G\left(\xi_{i}\right) \leq G\left(\xi_{j}\right)\right\}} \\
& +\frac{t-k^{*}}{n-k^{*}} \sum_{i=k^{*}+1}^{n} \sum_{j=1}^{k^{*}} 1_{\left\{G\left(\xi_{i}\right) \leq G\left(\xi_{j}\right)\right\}}+\frac{t-k^{*}}{n-k^{*}} \sum_{i=k^{*}+1}^{n} \sum_{j=k^{*}+1}^{n} 1_{\left\{G\left(\xi_{i}\right) \leq G\left(\xi_{j}\right)\right\}} \\
= & -\sum_{i=1}^{t} \sum_{j=t+1}^{n} 1_{\left\{G\left(\xi_{i}\right) \leq G\left(\xi_{j}\right)\right\}}-\frac{t(t+1)}{2}+\sum_{i=1}^{k^{*}} \sum_{j=k^{*}+1}^{n} 1_{\left\{G\left(\xi_{i}\right) \leq G\left(\xi_{j}\right)\right\}}+\frac{k^{*}\left(k^{*}+1\right)}{2} \\
& +\frac{t-k^{*}}{n-k^{*}} \sum_{i=k^{*}+1}^{n} \sum_{j=1}^{k^{*}}\left(1-1_{\left\{G\left(\xi_{j}\right) \leq G\left(\xi_{i}\right)\right\}}\right)+\frac{t-k^{*}}{n-k^{*}} \frac{\left(n-k^{*}\right)\left(n-k^{*}+1\right)}{2} \\
= & -\sum_{i=1}^{t} \sum_{j=t+1}^{n} 1_{\left\{G\left(\xi_{i}\right) \leq G\left(\xi_{j}\right)\right\}}-\frac{t(t+1)}{2}+\sum_{i=1}^{k^{*}} \sum_{j=k^{*}+1}^{n} 1_{\left\{G\left(\xi_{i}\right) \leq G\left(\xi_{j}\right)\right\}}+\frac{k^{*}\left(k^{*}+1\right)}{2} \\
& +\left(t-k^{*}\right) k^{*}-\frac{t-k^{*}}{n-k^{*}} \sum_{j=1}^{k^{*}} \sum_{i=k^{*}+1}^{n} 1_{\left\{G\left(\xi_{j}\right) \leq G\left(\xi_{i}\right)\right\}}+\frac{\left(t-k^{*}\right)\left(n-k^{*}+1\right)}{2} .
\end{aligned}
$$

Based on Lemma 1 and Corollary 1 , the argumentation that also established $\frac{1}{n^{2}} \hat{S}_{\lfloor n \lambda\rfloor}\left(1, k^{*}\right) \stackrel{P}{\longrightarrow} 0$ yields $\frac{1}{n^{2}} \hat{S}_{\lfloor n \lambda\rfloor}\left(k^{*}+1, n\right) \stackrel{P}{\longrightarrow} 0$ uniformly in $\lambda \geq \tau$.

Likewise, it can be shown that $\frac{1}{n^{2}} \tilde{S}_{\lfloor n \lambda\rfloor}\left(k^{*}+1, n\right) \stackrel{P}{\longrightarrow} 0$. First of all, we note that

$$
1_{\left\{G\left(\xi_{i}\right) \leq G\left(\xi_{j}\right)<G\left(\xi_{i}\right)+\Delta\right\}}=1_{\left\{G\left(\xi_{j}\right) \leq G\left(\xi_{i}\right)+\Delta\right\}}-1_{\left\{G\left(\xi_{j}\right) \leq G\left(\xi_{i}\right)\right\}}
$$

almost surely if $i \neq j$. Thereby,

$$
\begin{aligned}
\tilde{S}_{t}\left(k^{*}+1, n\right)= & \sum_{h=k^{*}+1}^{t}\left(\sum_{j=1}^{k^{*}} 1_{\left\{G\left(\xi_{h}\right) \leq G\left(\xi_{j}\right)<G\left(\xi_{h}\right)+\Delta\right\}}-\frac{1}{n-k^{*}} \sum_{i=k^{*}+1}^{n} \sum_{j=1}^{k^{*}} 1_{\left\{G\left(\xi_{i}\right) \leq G\left(\xi_{j}\right)<G\left(\xi_{i}\right)+\Delta\right\}}\right) \\
= & \sum_{j=1}^{k^{*}} \sum_{i=k^{*}+1}^{t} 1_{\left\{G\left(\xi_{j}\right) \leq G\left(\xi_{i}\right)+\Delta\right\}}-\frac{t-k^{*}}{n-k^{*}} \sum_{j=1}^{k^{*}} \sum_{i=k^{*}+1}^{n} 1_{\left\{G\left(\xi_{j}\right) \leq G\left(\xi_{i}\right)+\Delta\right\}} \\
& -\sum_{j=1}^{k^{*}} \sum_{i=k^{*}+1}^{t} 1_{\left\{G\left(\xi_{j}\right) \leq G\left(\xi_{i}\right)\right\}}+\frac{t-k^{*}}{n-k^{*}} \sum_{j=1}^{k^{*}} \sum_{i=k^{*}+1}^{n} 1_{\left\{G\left(\xi_{j}\right) \leq G\left(\xi_{i}\right)\right\}} .
\end{aligned}
$$

As a result, we have $\frac{1}{n^{2}} \tilde{S}_{\lfloor n \lambda\rfloor}\left(k^{*}+1, n\right) \stackrel{P}{\longrightarrow} 0$ by Lemma 2 and Corollary 1 .

As both terms, $\frac{1}{n^{2}} \hat{S}_{\lfloor n \lambda\rfloor}\left(k^{*}+1, n\right)$ as well as $\frac{1}{n^{2}} \tilde{S}_{\lfloor n \lambda\rfloor}\left(k^{*}+1, n\right)$, converge in probability to 0 uniformly in $\lambda \geq \tau$, it follows that

$$
\int_{\tau}^{1}\left\{\frac{1}{n^{2}} S_{\lfloor n r\rfloor}\left(k^{*}+1, n\right)\right\}^{2} d r \stackrel{P}{\rightarrow} 0 .
$$

On the basis of the previous considerations we may conclude that the denominator of $G_{n}\left(k^{*}\right)$ converges in probability to 0 . 
In order to prove the consistency of the self-normalized Wilcoxon change-point test, it therefore remains to show that the numerator of $G_{n}\left(k^{*}\right)$, given by

$$
\frac{1}{n^{2}}\left|\sum_{i=1}^{k^{*}} \sum_{j=k^{*}+1}^{n}\left(1_{\left\{X_{i} \leq X_{j}\right\}}-\frac{1}{2}\right)\right|
$$

converges to a non-negative constant.

We have

$$
\frac{1}{n^{2}}\left|\sum_{i=1}^{k^{*}} \sum_{j=k^{*}+1}^{n}\left(1_{\left\{X_{i} \leq X_{j}\right\}}-\frac{1}{2}\right)\right|=\left|\frac{1}{n^{2}} \sum_{i=1}^{k^{*}} \sum_{j=k^{*}+1}^{n} 1_{\left\{G\left(\xi_{i}\right) \leq G\left(\xi_{j}\right)+\Delta\right\}}-\frac{1}{n^{2}} \frac{k^{*}\left(n-k^{*}\right)}{2}\right| .
$$

Therefore,

$$
\frac{1}{n^{2}}\left|\sum_{i=1}^{k^{*}} \sum_{j=k^{*}+1}^{n}\left(1_{\left\{X_{i} \leq X_{j}\right\}}-\frac{1}{2}\right)\right| \stackrel{P}{\longrightarrow} \tau(1-\tau) \int_{\mathbb{R}}(F(x+\Delta)-F(x)) d F(x)
$$

by Corollary 1 and since $\frac{1}{n^{2}} \frac{k^{*}\left(n-k^{*}\right)}{2} \longrightarrow \frac{\tau(1-\tau)}{2}$. As the limit in 13 does not vanish, $G_{n}\left(k^{*}\right)$ diverges to $\infty$ and we thus have proved Theorem 2

Proof of Theorem 3. Note that because of the corresponding sample path properties of the stochastic process $Z$, the sample paths of

$$
W_{m, \tau}^{*}(\lambda)=(Z(\lambda)-\lambda Z(1)) \int_{\mathbb{R}} J(x) d F(x)+c \delta_{\tau}(\lambda) \int_{\mathbb{R}} f^{2}(x) d x, 0 \leq \lambda \leq 1,
$$

are almost surely continuous and nowhere differentiable.

The same argument as in the proof of Theorem 1 shows that $T_{n}\left(\tau_{1}, \tau_{2}\right)$ converges in distribution to

$$
\sup _{\lambda \in\left[\tau_{1}, \tau_{2}\right]} \frac{\left|W_{m, \tau}^{*}(\lambda)\right|}{\left\{\int_{0}^{\lambda}\left(W_{m, \tau}^{*}(r)-\frac{r}{\lambda} W_{m, \tau}^{*}(\lambda)\right)^{2} d r+\int_{\lambda}^{1}\left(W_{m, \tau}^{*}(r)-\frac{1-r}{1-\lambda} W_{m, \tau}^{*}(\lambda)\right)^{2} d r\right\}^{\frac{1}{2}} .}
$$

The numerator of the limit process equals

$$
\left|\int_{\mathbb{R}} J(x) d F(x)(Z(\lambda)-\lambda Z(1))+c \delta_{\tau}(\lambda) \int_{\mathbb{R}} f^{2}(x) d x\right| .
$$

Moreover, for the quantities in the denominator it holds that

$$
\begin{aligned}
W_{m, \tau}^{*}(r)-\frac{r}{\lambda} W_{m, \tau}^{*}(\lambda)= & (Z(r)-r Z(1)) \int_{\mathbb{R}} J(x) d F(x)+c \delta_{\tau}(r) \int_{\mathbb{R}} f^{2}(x) d x \\
& -\frac{r}{\lambda}\left((Z(\lambda)-\lambda Z(1)) \int_{\mathbb{R}} J(x) d F(x)+c \delta_{\tau}(\lambda) \int_{\mathbb{R}} f^{2}(x) d x\right) \\
= & \int_{\mathbb{R}} J(x) d F(x)\left(Z(\lambda)-\frac{r}{\lambda} Z(\lambda)\right)+c \int_{\mathbb{R}} f^{2}(x) d x\left(\delta_{\tau}(r)-\frac{r}{\lambda} \delta_{\tau}(\lambda)\right)
\end{aligned}
$$

and

$$
\begin{aligned}
W_{m, \tau}^{*}(r)-\frac{1-r}{1-\lambda} W_{m, \tau}^{*}(\lambda)= & (Z(r)-r Z(1)) \int_{\mathbb{R}} J(x) d F(x)+c \delta_{\tau}(r) \int_{\mathbb{R}} f^{2}(x) d x \\
& -\frac{1-r}{1-\lambda}\left\{(Z(\lambda)-\lambda Z(1)) \int_{\mathbb{R}} J(x) d F(x)+c \delta_{\tau}(\lambda) \int_{\mathbb{R}} f^{2}(x) d x\right\} \\
= & \int_{\mathbb{R}} J(x) d F(x)\left\{Z(r)-r Z(1)-\frac{1-r}{1-\lambda}(Z(\lambda)-\lambda Z(1))\right\} \\
& +c \int_{\mathbb{R}} f^{2}(x) d x\left(\delta_{\tau}(r)-\frac{1-r}{1-\lambda} \delta_{\tau}(\lambda)\right) .
\end{aligned}
$$




\section{References}

Andrews, D. W. K. (1993) Tests for Parameter Instability and Structural Change with Unknown Change Point. Econometrica 61, 821-856.

Dehling, H. G., Rooch, A., and Taqqu, M. S. (2013a) Non-Parametric Change-Point Tests for Long-Range Dependent Data. Scandinavian Journal of Statistics 40, 153 - 173.

Dehling, H. G., Rooch, A., and Taqqu, M. S. (2013b) Power of Change-Point Tests for Long-Range Dependent Data. arXiv:1303.4917.

Dehling, H. G. and Taqqu, M. S. (1989) The Empirical Process of some Long-Range Dependent Sequences with an Application to U-Statistics. The Annals of Statistics 17, 1767-1783.

Krengel, U. and Brunel, A. (1985) Ergodic theorems. Walter de Gruyter Berlin, New York.

Lobato, I. N. (2001) Testing That a Dependent Process Is Uncorrelated. Journal of the American Statistical Association 96, 1066-1076.

Maejima, M. and Tudor, C. A. (2007) Wiener integrals with respect to the Hermite process and a NonCentral Limit Theorem. Stochastic Analysis and Applications 25, 1043-1056.

Mikosch, T. (1998) Elementary Stochastic Calculus With Finance in View. World Scientific Publishing Co. Pte. Ltd.

Shao, X. (2011) A simple test of changes in mean in the possible presence of long-range dependence. Journal of Time Series Analysis 32(6), 598-606.

Shao, X. and Zhang, X. (2010) Testing for Change Points in Time Series. Journal of The American Statistical Association 105, 1228-1240.

Shorack, G. R. and Wellner, J. A. (1986) Empirical Processes with Applications to Statistics. John Wiley \& Sons, New York.

Taqqu, M. S. (1979) Convergence of Integrated Processes of Arbitrary Hermite Rank. Zeitschrift für Wahrscheinlichkeitstheorie und verwandte Gebiete 50, 53-83. 